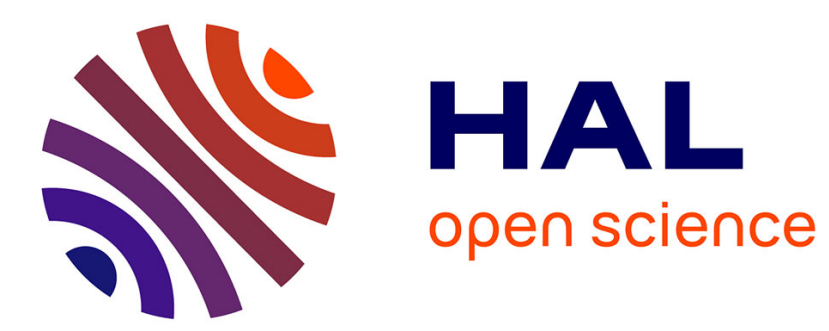

\title{
Total Variation as a local filter
}

Cécile Louchet, Lionel Moisan

\section{- To cite this version:}

Cécile Louchet, Lionel Moisan. Total Variation as a local filter. SIAM Journal on Imaging Sciences, 2011, 4 (2), pp.651-694. 10.1137/100785855 . hal-00457763

\section{HAL Id: hal-00457763 https://hal.science/hal-00457763}

Submitted on 18 Feb 2010

HAL is a multi-disciplinary open access archive for the deposit and dissemination of scientific research documents, whether they are published or not. The documents may come from teaching and research institutions in France or abroad, or from public or private research centers.
L'archive ouverte pluridisciplinaire HAL, est destinée au dépôt et à la diffusion de documents scientifiques de niveau recherche, publiés ou non, émanant des établissements d'enseignement et de recherche français ou étrangers, des laboratoires publics ou privés. 


\title{
Total Variation as a local filter
}

\author{
Cécile Louchet \\ Université d'Orléans \\ MAPMO, CNRS UMR 6628 \\ Cecile.Louchet@univ-orleans.fr
}

\author{
Lionel Moisan
}

\author{
Université Paris Descartes \\ MAP5, CNRS UMR 8145 \\ Lionel.Moisan@parisdescartes.fr
}

\begin{abstract}
In the Rudin-Osher-Fatemi (ROF) image denoising model, Total Variation (TV) is used as a global regularization term. However, as we observe, the local interactions induced by Total Variation do not propagate much at long distances in practice, so that the ROF model is not far from being a local filter. In this paper, we propose to build a purely local filter by considering the ROF model in a given neighborhood of each pixel. We show that appropriate weights are required to avoid aliasinglike effects, and we provide an explicit convergence criterion for an associated dual minimization algorithm based on Chambolle's work. We study theoretical properties of the obtained local filter, and show that this localization of the ROF model brings an interesting optimization of the biasvariance trade-off, and a strong reduction a ROF drawback called "staircasing effect". We finally present a new denoising algorithm, TV-means, that efficiently combines the idea of local TV-filtering with the non-local means patch-based method.
\end{abstract}

Keywords : Total variation, variational model, local filter, image denoising, non-local means.

\section{Introduction}

Image denoising/smoothing is one of the most considered issues in image processing, not only because it plays a key preliminary role in many computer vision systems, but also because it is probably the simplest way to address the fundamental issue of image modeling, as a starting point towards more complex tasks like deblurring, demosaicking, inpainting, etc. Among denoising/smoothing methods, several classes arise naturally. One of them consists in local filters, that is, translation-invariant operators that transform a gray-level image $v: \Omega \rightarrow \mathbb{R}$ into a gray-level image

$$
u: x \mapsto T\left((v(x+z))_{z \in B}\right),
$$

where $B$ a bounded set (typically, a disc with radius $r$ centered in 0 ), and $T$ an application from $\mathbb{R}^{B}$ to $\mathbb{R}$. Note that this definition equally holds for images defined on a continuous domain $\left(\Omega, B \subset \mathbb{R}^{2}\right)$ or on a discrete domain $\left(\Omega, B \subset \mathbb{Z}^{2}\right)$. Among local filters are the averaging filter, obtained (in a continuous setting) with the averaging operator

$$
T(w)=\frac{1}{|B|} \int_{B} w(z) d z
$$

or more generally convolution filters with finite impulse response (when $T$ is linear). Let us also mention contrast-invariant operators like, for example, the median filter, or the erosion filter associated to

$$
T(w)=\inf _{z \in B} w(z) .
$$


Another important example is given by Yaroslavsky's filter [60], corresponding to

$$
T(w)=\frac{1}{C(w)} \int_{B} w(z) e^{-|w(z)-w(0)|^{2} / h^{2}} d z, \quad \text { where } \quad C(w)=\int_{B} e^{-|w(z)-w(0)|^{2} / h^{2}} d z
$$

and the related (not strictly local) SUSAN [56] and bilateral filters [58] (see [16] for a discussion on the relationship between these three filters).

Another class of denoising/smoothing methods consists in variational formulations, which transform an image $v$ into an image $u$ that minimizes some energy functional $E_{\lambda}(u)$ depending on $v$ and on a parameter $\lambda$. A typical example is the $L^{2}-H^{1}$ minimization, associated to

$$
E_{\lambda}(u)=\|u-v\|^{2}+\lambda \int_{\Omega}|\nabla u|^{2}
$$

where $\nabla u=\left(\frac{\partial u}{\partial x}, \frac{\partial u}{\partial y}\right)^{T}$ is the gradient of $u$. This example is a special case of the Wiener filter, that can be solved explicitly when $\Omega=\mathbb{R}^{2}$ with

$$
\forall \xi \in \mathbb{R}^{2}, \quad \hat{u}(\xi)=\frac{\hat{v}(\xi)}{1+\lambda|\xi|^{2}}
$$

where $\hat{f}$ denotes the Fourier transform of $f: \mathbb{R}^{2} \rightarrow \mathbb{R}$. This filter is a convolution, but since the associated kernel is not compactly supported, it cannot be written as a purely local filter. A more sophisticated example, avoiding undesirable blur effects caused by (1), is obtained with

$$
E_{\lambda}(u)=\|u-v\|^{2}+\lambda \int_{\Omega}|\nabla u|
$$

which corresponds to the Rudin-Osher-Fatemi (ROF) model for image denoising [54]. This model, which is the starting point of this work, has been widely used in image processing for various tasks including denoising [24, 36], deblurring [23, 55], interpolation [40], super-resolution [3, 21], inpainting [25, 26], cartoon/texture decomposition [8,51], etc. Dramatic improvements have also been recently made on accuracy and computation speed for the numerical solving of ROF-derived variational problems $[9,17$, $21,22,33,34,59]$.

These two classes of methods (not to mention others) correspond to two different points of view. A local filter can be iterated, which generally results in a Partial Differential Equation (PDE) formulation with interesting interpretations (the heat equation for positive isotropic averaging filters, the mean curvature motion for the median filter [5], etc.). The amount of smoothing/denoising can also be increased by changing the size of the neighborhood $B$, whereas in a variational formulation (energy $E_{\lambda}$ ), this role is played by the hyperparameter $\lambda$ that controls the trade-off between the smoothness of $u$ and its distance to the original image $v$. The use of local filters is very natural in a shape recognition context, where the possibility of occlusions makes long-distance smoothing interactions questionable (it seems more relevant to smooth the background of a scene mostly independently of the foreground, and vice-versa). In general, variational formulations do not lead to local filters, because short-distance interactions involved in $E_{\lambda}(u)$ (typically resulting from partial derivatives) cause long-distance interactions due to the minimization process $[12]$.

In this work, we first study the importance of these long-distance interactions for the ROF model (Equation 2). We show in Section 2 that most image pixels have a very limited influence zone around them, which suggests that the ROF model is not far from being a local filter. In Section 3, we follow this idea and derive a local filter by considering a ROF model on a neighborhood of each pixel. We show in particular that the introduction of a smooth window (that is, appropriate weights on the neighborhood) is required to avoid aliasing-like artifacts (that is, the enhancement of particular frequencies). The monotony of this local TV-filter is investigated in Section 4, and we show that it admits two limiting PDEs: the Total variation flow [10] and the heat equation. In Section 5, we build a numerical scheme inspired from 
Chambolle's algorithm [21] to solve the weighted local ROF model on an arbitrary neighborhood, and give a convergence criterion involving a relationship between the time step and the weighting function. Experiments performed in Section 6 show in particular two interesting properties of the local TV-filter compared to the global ROF model: its ability to reach an intermediate bias-variance trade-off for image denoising, and the elimination of a well-known artifact called "staircasing effect". To illustrate the perspectives offered by local TV-filtering, we build in Section 7 a new denoising filter, called $T V$-means, that combines in a simple way the strengths of TV-denoising and NL-means denoising [15], and produces much better results than both of them.

\section{How nonlocal is TV-denoising?}

In this section we investigate the amount of locality of the ROF denoising model. As we shall see, even though TV-denoising requires a global optimization on the whole image, local interactions do not propagate very far, and the gray level of a denoised pixel essentially depends on the pixels lying in its neighborhood, while other pixels have negligible or null impact.

In the following, a (discrete) image is a function $u: \Omega \rightarrow \mathbb{R}$, where $\Omega$ is a subset of $\mathbb{Z}^{2}$ (the set of pixels) and $u(x, y)$ represents the gray-level at pixel $(x, y)$. If $A$ is subset of $\mathbb{Z}^{2}, A^{c}$ will denote its complement, and $\partial A$ is the boundary of $A$, defined as the set of pixels for which at least one neighbor (for the 4-neighbor topology) does not belong to $A$. To a subset $A$ of $\mathbb{Z}^{2}$ we associate the set $A^{\square} \subset \mathbb{R}^{2}$ defined by

$$
A^{\square}=\bigcup_{(i, j) \in A}\left[i-\frac{1}{2}, i+\frac{1}{2}\right] \times\left[j-\frac{1}{2}, j+\frac{1}{2}\right] .
$$

Thus, $A^{\square}$ is obtained by considering grid points of $\mathbb{Z}^{2}$ as $1 \times 1$ squares of $\mathbb{R}^{2}$. It is interesting to notice that if a discrete image $u: \mathbb{Z}^{2} \rightarrow \mathbb{R}$ is extended to an image $\bar{u}: \mathbb{R}^{2} \rightarrow \mathbb{R}$ using the nearest neighbor interpolation, then the level sets of $\bar{u}$ are obtained by applying the ${ }^{\square}{ }^{\circ}$ operator to the level sets of $u$, that is,

$$
\forall \lambda \in \mathbb{R}, \quad\left\{x \in \mathbb{R}^{2}, \bar{u}(x) \leq \lambda\right\}=\left\{x \in \mathbb{Z}^{2}, u(x) \leq \lambda\right\}^{\square} .
$$

The ${ }^{\square}$ operator allows to extend the usual Perimeter operator (defined on Caccioppoli sets of $\mathbb{R}^{2}$ ) to discrete sets, with

$$
\forall A \subset \mathbb{Z}^{2}, \quad \operatorname{per} A=\operatorname{Perimeter}\left(A^{\square}\right) .
$$

Let us first recall the principle of ROF denoising [54]. If $u$ is an image defined on $\Omega \subset \mathbb{Z}^{2}$, its Total Variation (TV) is defined by

$$
T V(u)=\sum_{(i, j) \in \Omega}|\nabla u(i, j)|,
$$

where $|\nabla u(i, j)|$ denotes a given scheme of the gradient norm of $u$ at pixel $(i, j)$. If $v$ is a noisy image, the ROF model proposes to smooth it by selecting the unique image $u=T(v)$ that minimizes

$$
E_{\lambda}(u)=\|u-v\|^{2}+\lambda T V(u),
$$

where $\|\cdot\|$ stands for the classical Euclidean norm on $\mathbb{R}^{\Omega}$. The positive parameter $\lambda$ controls the amount of denoising and should be set accordingly to the noise level.

In the sequel, as in [21], we shall choose for $\nabla u(i, j)$ a scheme based on simple differences between neighbor pixels, that is

$(\nabla u)_{i, j}^{1}=\left\{\begin{array}{ll}u(i+1, j)-u(i, j) & \text { if }(i+1, j) \in \Omega \\ 0 & \text { else }\end{array} \quad\right.$ and $(\nabla u)_{i, j}^{2}= \begin{cases}u(i, j+1)-u(i, j) & \text { if }(i, j+1) \in \Omega \\ 0 & \text { else, }\end{cases}$

and we shall derive $|\nabla u(i, j)|$ by considering either the $\ell^{1}$-norm or the $\ell^{2}$-norm of $\nabla u(i, j)$ in $\mathbb{R}^{2}$. Each choice involves its own specificities : 
- the $\ell^{1}$-norm of the gradient, given by

$$
|\nabla u(i, j)|_{1}=\left|(\nabla u)_{i, j}^{1}\right|+\left|(\nabla u)_{i, j}^{2}\right|
$$

is not isotropic (it favors vertical and horizontal directions), but has the advantage of making $T V$ satisfy the coarea formula $[22,33,38]$

$$
\forall u \in \mathbb{R}^{\Omega}, \quad T V(u)=\int_{\mathbb{R}} \operatorname{per}\{x \in \Omega ; u(x) \leq \lambda\} d \lambda,
$$

which allows to interpret $T V(u)$ as the cumulated length of the level lines of $u$.

Furthermore, ROF denoising in that case is monotone, in the sense that

$$
\left[\forall x \in \Omega, v_{1}(x) \leq v_{2}(x)\right] \Longrightarrow\left[\forall x \in \Omega, T\left(v_{1}\right)(x) \leq T\left(v_{2}\right)(x)\right]
$$

(see [22] for a proof). In this section, these analytic properties will be most useful to study the locality of TV-denoising; this is why we will consider the $\ell^{1}$-norm.

- the $\ell^{2}$-norm of the gradient, given by

$$
|\nabla u(i, j)|_{2}=\sqrt{\left((\nabla u)_{i, j}^{1}\right)^{2}+\left((\nabla u)_{i, j}^{2}\right)^{2}}
$$

is more isotropic, and in practice, the images denoised with this scheme look slightly more natural. However neither the coarea formula nor the monotony principle (see Section 4 ) hold any more. This $\ell^{2}$-norm will be used in the numerical experiments (Section 6).

Both $\ell^{1}$ and $\ell^{2}$ schemes are compatible with two other quite basic properties of operator $T$. Namely, they force the TV-denoising operator to preserve the image average, i.e.

$$
\forall v \in \mathbb{R}^{\Omega}, \quad \sum_{x \in \Omega} T(v)(x)=\sum_{x \in \Omega} v(x)
$$

and to be shift invariant, i.e.

$$
\forall v \in \mathbb{R}^{\Omega}, \forall b \in \mathbb{R}, \quad T(v+b)=T(v)+b,
$$

which will be useful in the sequel.

Let us now discuss the locality of TV-denoising, that is, the influence region of an arbitrary pixel in the denoising process. We easily observe that the dependence in $E_{\lambda}$ of a gray level $u(x)$ brings into play the pixels $y$ that are neighbors of $x$ only, through their gray levels $u(y)$. But the levels $u(y)$ also depend on their neighbors and so on, such that the denoising is likely to be indeed global, in the sense that a change on a pixel in the noisy image might change the value of any other pixel in the denoised image.

For instance, let $\Omega$ be a rectangular domain, and $x_{0}$ be a pixel in $\Omega \backslash \partial \Omega$. If the image $v$ is an impulse image (discrete Dirac) defined for some $A \in \mathbb{R}$ by

$$
\forall x \in \Omega, \quad v(x)=A \cdot \delta_{x=x_{0}}=\left\{\begin{array}{lll}
A & \text { if } & x=x_{0}, \\
0 & \text { else, }
\end{array}\right.
$$

(here $\delta$ is the $0-1$ Kronecker-delta function), then simple calculations show that $T(v)$, computed with the $\ell^{1}$-scheme, satisfies

$$
\forall x \in \Omega, \quad T(v)(x)= \begin{cases}(A-2 \lambda) \delta_{x=x_{0}}+\frac{2 \lambda}{|\Omega|-1} \delta_{x \neq x_{0}} & \text { if } A>2 \lambda \\ A /|\Omega| & \text { if }|A| \leq 2 \lambda \\ (A+2 \lambda) \delta_{x=x_{0}}-\frac{2 \lambda}{|\Omega|-1} \delta_{x \neq x_{0}} & \text { if } A<-2 \lambda\end{cases}
$$


where $|\Omega|$ denotes the cardinal of $\Omega$. This means that any pixel of the domain can be affected by a change in $v\left(x_{0}\right)$. However, one can notice that when $|\Omega|$ is large, then the change in any pixel $x$ is negligible with respect to the change of $x_{0}$; hence the globality of TV-denoising is rather weak in this example. Note also that if $\Omega$ is finite, the scheme (5) corresponds to Neumann conditions in the minimization of (4), because the gradient at a pixel lying on the boundary $\partial \Omega$ is treated as if its facing neighbors lying outside $\Omega$ had the same gray level. TV-denoising based on these Neumann conditions has the property of preserving the image average (10), which explains the slight correction of $\pm 2 \lambda /(|\Omega|-1)$ obtained on the pixels $x \neq x_{0}$ in $(13)$.

\subsection{Locality and boundary conditions}

In the sequel of Section 2, we shall bring evidence to the fact that, with appropriate boundary conditions, TV-denoising is essentially local, both in exact and in simulated examples. We propose here to consider boundary conditions other than Neumann conditions. First we consider infinite boundary conditions: an image is continued by 0 on the entire set $\mathbb{Z}^{2}$, and is viewed as finitely supported function, that is a function $u$ whose support

$$
\operatorname{supp}(u)=\{x \in \Omega, u(x) \neq 0\}
$$

is a finite set.

Proposition 1 (Locality for an infinite domain) Let $v \in \ell^{2}\left(\mathbb{Z}^{2}\right)$. We consider the variational problem

$$
\text { minimize } \sum_{x \in \mathbb{Z}^{2}}(u(x)-v(x))^{2}+\lambda T V(u) \quad \text { for } u \in \ell^{2}\left(\mathbb{Z}^{2}\right),
$$

where $T V(u)$ is computed according to the $\ell^{1}$-scheme (6) with $\Omega=\mathbb{Z}^{2}$. If $v$ has a finite support, i.e. if there exists a rectangle $\Omega_{0} \subset \mathbb{Z}^{2}$ satisfying $\operatorname{supp}(v) \subset \Omega_{0}$, then the image $T(v)$ solving the variational problem (14) is also finitely supported and satisfies $\operatorname{supp}(T(v)) \subset \Omega_{0}$.

Proof -1) Let $A \geq 0$ and $v=A \mathbb{1}_{\Omega_{0}}$, where $\Omega_{0}$ is any rectangle of $\mathbb{Z}^{2}$, and where

$$
\forall x \in \mathbb{Z}^{2}, \quad \mathbb{1}_{\Omega_{0}}(x)= \begin{cases}1 & \text { if } x \in \Omega_{0} \\ 0 & \text { otherwise }\end{cases}
$$

We prove $\operatorname{supp}(T(v)) \subset \Omega_{0}$ by showing that $\left\{x \in \mathbb{Z}^{2}, T(v)(x)>0\right\} \subset \Omega_{0}$ and then that $T(v)$ is nonnegative. Assume that the upper level set $\left\{x \in \mathbb{Z}^{2}, T(v)(x)>0\right\}$, shortly written $\{T(v)>0\}$, intersects the complement $\Omega_{0}^{c}$ of $\Omega_{0}$. Consider the image $w=T(v) \cdot \mathbb{1}_{\Omega_{0}}$. Changing $T(v)$ into $w$ decreases the data-fidelity term because

$$
\sum_{x \in \mathbb{Z}^{2}}(w(x)-v(x))^{2}=\sum_{x \in \Omega_{0}}(T(v)(x)-v(x))^{2}<\sum_{x \in \mathbb{Z}^{2}}(T(v)(x)-v(x))^{2} .
$$

It also decreases the Total Variation. Indeed a level set $\{w \geq \lambda\}$ satisfies

$$
\{w \geq \lambda\}=\{T(v) \geq \lambda\} \cap \Omega_{0},
$$

and thanks to the convexity of $\Omega_{0}^{\square}$ we get

$$
\operatorname{per}\{w \geq \lambda\}=\operatorname{per}\left(\{T(v) \geq \lambda\} \cap \Omega_{0}\right)=\operatorname{Perimeter}\left(\{T(v) \geq \lambda\}^{\square} \cap \Omega_{0}^{\square}\right) \leq \operatorname{per}\{T(v) \geq \lambda\} .
$$

Hence thanks to the coarea formula (7), we get

$$
T V(w)=\int_{\mathbb{R}} \operatorname{per}\{w \geq \lambda\} d \lambda<\int_{\mathbb{R}} \operatorname{per}\{T(v) \geq \lambda\} d \lambda=T V(T(v)) .
$$


Finally we get

$$
E_{\lambda}(w)=\sum_{x \in \mathbb{Z}^{2}}(w(x)-v(x))^{2}+\lambda T V(w) \leq \sum_{x \in \mathbb{Z}^{2}}(T(v)(x)-v(x))^{2}+\lambda T V(T(v))=E_{\lambda}(T(v)),
$$

and we obtain a contradiction to the optimality of $T(v)$.

Now assume that the lower level set $\{T(v)<0\}$ is non-empty. We consider the positive part $T(v)_{+}$ of $T(v)$, where $(y)_{+}=\max (y, 0)$ for any $y$. As before, we have $\left\|T(v)_{+}-v\right\|^{2}<\|T(v)-v\|^{2}$ and

$$
T V\left(T(v)_{+}\right)=\int_{\mathbb{R}^{+}} \operatorname{per}(\{T(v)<\lambda\}) d \lambda \leq \int_{\mathbb{R}} \operatorname{per}(\{T(v)<\lambda\}) d \lambda=T V(T(v)) .
$$

This contradicts the optimality of $T(v)$ and proves that $\{T(v)<0\}$ is empty. The proposition is therefore proven for $v=A \mathbb{1}_{\Omega_{0}}$.

2) Now if $v$ is an arbitrary image satisfying the conditions of the proposition, then

$$
-A \mathbb{1}_{\Omega_{0}} \leq v \leq A \mathbb{1}_{\Omega_{0}} \quad \text { where } \quad A=\max _{x \in \Omega_{0}}|v(x)| .
$$

By monotony of TV-denoising (see Equation 8), the inequalities remain true for the denoised images, i.e.

$$
-T\left(A \mathbb{1}_{\Omega_{0}}\right) \leq T(v) \leq T\left(A \mathbb{1}_{\Omega_{0}}\right),
$$

where the leftmost and rightmost terms both have their support included in $\Omega_{0}$, thanks to 1 ). Consequently, $\operatorname{supp}(T(v)) \subset \Omega_{0}$, as announced.

Now we consider Dirichlet boundary conditions, that impose that the denoised image vanishes on $\partial \Omega$. Then the same kind of result holds, as shown by the following corollary.

Corollary 1 (Locality for Dirichlet boundary conditions) Let $\Omega$ be a subset of $\mathbb{Z}^{2}$, and $v: \Omega \rightarrow \mathbb{R}$. We consider the variational problem

$$
\text { minimize } \sum_{x \in \Omega}(u(x)-v(x))^{2}+\lambda T V(u) \quad \text { subject to } \quad \forall x \in \partial \Omega, u(x)=0 .
$$

If there exists a rectangle $\Omega_{0}$ satisfying $\operatorname{supp}(v) \subset \Omega_{0} \subset \Omega \backslash \partial \Omega$, then the image $T(v)$ solving the variational problem (16) satisfies $\operatorname{supp}(T(v)) \subset \Omega_{0}$.

Proof — Let us denote by $\dot{v}$ the image $v$ continued by 0 on the plane $\mathbb{Z}^{2}$. If $u$ is the solution of (16), then $u$ is also the solution of the following problem: minimize $E_{\lambda}(u)$ for $u \in \ell^{2}\left(\mathbb{Z}^{2}\right)$ under the constraint

$$
\forall x \in\left(\mathbb{Z}^{2} \backslash \Omega\right) \cup \partial \Omega, \quad u(x)=0,
$$

with $E_{\lambda}(u)=\sum_{x \in \mathbb{Z}^{2}}(u(x)-\dot{v}(x))^{2}+\lambda T V(u)$. Now consider the solution $u^{\prime}=T(v)$ of Problem (14), that is, the minimizer of $E_{\lambda}$ over $\ell^{2}\left(\mathbb{Z}^{2}\right)$. Since $E_{\lambda}\left(u^{\prime}\right) \leq E_{\lambda}(\dot{u})$ and $u^{\prime}$ satisfies (17) thanks to Proposition 1 (because $v=0$ on $\left.\left(\mathbb{Z}^{2} \backslash \Omega\right) \cup \partial \Omega \supset \Omega_{0}^{c}\right)$, we necessarily have $u^{\prime}=\dot{u}$ and consequently $\operatorname{supp}(u)=\operatorname{supp}(\dot{u})=$ $\operatorname{supp}\left(u^{\prime}\right) \subset \Omega_{0}$.

A consequence of the two results above is that in both cases (infinite domain or Dirichlet boundary conditions), the TV-denoising of the impulse image (12) remains an impulse image, and the pixels apart from $x_{0}$ never change. More generally, if $v$ is finitely supported, then the influence of a pixel $x \in \operatorname{supp}(v)$ is limited to a rectangle $\Omega_{0}$ (the smallest rectangle containing $\operatorname{supp}(v)$ ), in the sense that for any $y \in \Omega_{0}^{c}$, $T(v)(y)$ does not depend on $v(x)$. This is a first result in favor of the locality of TV-denoising. 


\subsection{Locality for explicit solutions (continuous domain)}

Literature about exact solutions of TV-denoising in its continuous version (i.e. when $\Omega$ is an open subset of $\mathbb{R}^{2}$ ) provides valuable examples of locality in TV-denoising. First, Strong and Chan [57] provide exact solutions to the problem stated in one dimension and also in two dimensions for radially symmetric images, for either piecewise constant images, or for piecewise constant images with little noise added and small level of denoising $\lambda$. In each of these cases, it is notable that changing the (constant) value of a region can have repercussions only on this region and on its immediate neighboring regions. The propagation of the values change cannot go further.

Another case, more interesting here, is treated in [10]. The authors still assume that the images are defined on a continuous space, and consider an image $v$ writing as a linear combination of characteristic functions of convex sets $C_{i}$, i.e.

$$
v=\sum_{i=1}^{n} b_{i} \mathbb{1}_{C_{i}},
$$

where $\left(b_{i}\right)_{1 \leq i \leq n}$ is a sequence of arbitrary real numbers, the sets $C_{i}$ are assumed to be regular enough and spaced out enough (see [10] for the exact technical assumptions), and each function $\mathbb{1}_{C_{i}}$ is defined as in (15). Then they prove that the associated denoised image writes

$$
T(v)=\sum_{i=1}^{n} f_{C_{i}}\left(b_{i}\right) \mathbb{1}_{C_{i}}
$$

where $f_{C_{i}}$ is a soft-thresholding function (i.e. an odd function defined by $f_{C_{i}}(x)=\max \left(x-\tau_{C_{i}}, 0\right)$ for $x \geq 0$ ) whose threshold $\tau_{C_{i}}$ only depends on $C_{i}$. This means that for such images, the convex sets $\left(C_{i}\right)$ evolve independently of each other.

As the operator $T$ is monotone in this continuous framework (see [22] and references therein), we can state a more general property : assume that the image $v$ is null outside convex sets $C_{i}$, i.e.

$$
v=\sum_{i=1}^{n} v_{i}
$$

where each $v_{i}$ is a bounded image supported by $C_{i}$, and the $C_{i}$ 's are regular and spaced out enough as in (18) [10]. The monotony of $T$ implies

$$
T\left(\sum_{i=1}^{n}\left(\min v_{i}\right) \mathbb{1}_{C_{i}}\right) \leq T(v) \leq T\left(\sum_{i=1}^{n}\left(\max v_{i}\right) \mathbb{1}_{C_{i}}\right),
$$

where both leftmost and rightmost terms are exactly computable because the arguments of $T$ are in the general form (18). Then $T(v)$ satisfies

$$
\sum_{i=1}^{n} f_{C_{i}}\left(\min v_{i}\right) \mathbb{1}_{C_{i}} \leq T(v) \leq \sum_{i=1}^{n} f_{C_{i}}\left(\max v_{i}\right) \mathbb{1}_{C_{i}}
$$

which ensures that $T(v)$ vanishes outside from the sets $C_{i}$. Hence the minimization of the ROF energy relative to $v$ holds on the set of images $u$ writing $u=\sum_{i=1}^{n} u_{i}$ with $\operatorname{supp}\left(u_{i}\right) \subset C_{i}$. On such images, the ROF energy can be decomposed into

$$
\|u-v\|^{2}+\lambda T V(u)=\sum_{i=1}^{n}\left(\left\|u_{i}-v_{i}\right\|^{2}+\lambda T V\left(u_{i}\right)\right),
$$

that is a sum of independent ROF energies, showing that a change in $v_{i}$ will only affect the component of the energy corresponding to $C_{i}$, and that $T(v)$ will then be changed on $C_{i}$ at most : the components of $v$ evolve independently. 


\subsection{Locality for natural images : influence map of a pixel}

Here we focus on real-life natural images, and show locality properties of TV-denoising. To investigate the locality issue precisely, we shall say that a pixel $y$ is influenced by a pixel $x$ if $T(v)(y)$ depends on $v(x)$, and we measure this dependency by

$$
C_{x}(y)=\sup _{\delta \neq 0}\left|\frac{T\left(v+\delta \mathbb{1}_{\{x\}}\right)(y)-T(v)(y)}{\delta}\right| .
$$

This number measures the maximum relative impact on $T(v)(y)$ caused by a distortion of $v(x)$. Several properties of the influence map $C_{x}$ are gathered in the following

Theorem 1 (Properties of the influence map) Let $\Omega$ be a bounded subset of $\mathbb{Z}^{2}$, and $v: \Omega \rightarrow \mathbb{R}$. If $T V$ is computed with the $\ell^{1}$-scheme (6) and $T$ is the associated $T V$-denoising operator, then for any $x \in \Omega$, the influence map $C_{x}$ defined by (19) satisfies

(1) $C_{x}(x)=1$,

(2) for every $y \neq x, C_{x}(y)<1$.

(3) for any $\delta \neq 0$,

$$
\sum_{y \in \Omega}\left(\frac{T\left(v+\delta \mathbb{1}_{\{x\}}\right)(y)-T(v)(y)}{\delta}\right)^{2} \leq 1
$$

Theorem 1 tells us that a distortion of $v(x)$ cannot be amplified by $T$, and that it is indeed attenuated for all pixels except $x$ (as we shall see in the experiments, $C_{x}(y)$ decreases very quickly when $y$ goes away from $x$ ). Property (3) does not bring a direct estimate of the total influence of a pixel $x$, but shows that any given distortion $\delta$ on $v(x)$ causes a total distortion on $T(v)$ smaller than $\delta$ (in $\ell^{2}$-norm), since

$$
\left\|T\left(v+\delta \mathbb{1}_{\{x\}}\right)-T(v)\right\|_{2} \leq \delta .
$$

Proof of Theorem 1 Let $x \in \Omega$. For every $\delta \in \mathbb{R}$, we set $\hat{u}_{\delta}=T\left(v+\delta \mathbb{1}_{\{x\}}\right)$, so that

$$
C_{x}(y)=\sup _{\delta \neq 0}\left|\left(\hat{u}_{\delta}(y)-\hat{u}_{0}(y)\right) / \delta\right| .
$$

We first prove that for every $y \in \Omega, C_{x}(y) \leq 1$. Indeed, if $\delta \neq 0$,

$$
v-|\delta| \leq v+\delta \mathbb{1}_{\{x\}} \leq v+|\delta|,
$$

and the monotony of $T$, combined with shift invariance (11), implies that

$$
\hat{u}_{0}-|\delta| \leq \hat{u}_{\delta} \leq \hat{u}_{0}+|\delta| \text {. }
$$

Hence for any pixel $y \in \Omega$,

$$
\left|\frac{\hat{u}_{\delta}(y)-\hat{u}_{0}(y)}{\delta}\right| \leq 1
$$

and $C_{x}(y) \leq 1$ by considering the supremum on $\delta$.

Now we come to the very proof of the theorem, beginning with item (1), then (3) and finally (2).

(1) The Euler equation corresponding to the TV-denoising of image $v+\delta \mathbb{1}_{\{x\}}$ is

$$
\hat{u}_{\delta}-\left(v+\delta \mathbb{1}_{\{x\}}\right)+\frac{\lambda}{2} \partial T V\left(\hat{u}_{\delta}\right) \ni 0,
$$


where $\partial T V$ denotes the subdifferential of $T V$, defined by

$$
w \in \partial T V(u) \Longleftrightarrow \forall v \in \mathbb{R}^{\Omega}, \quad T V(v) \geq T V(u)+\langle w, v-u\rangle
$$

(see [41] for instance). Let sign be the set-valued function defined by

$$
\operatorname{sign}(x)= \begin{cases}\{1\} & \text { if } x>0, \\ \{-1\} & \text { if } x<0, \\ {[-1,1]} & \text { if } x=0\end{cases}
$$

The subdifferential of $T V$, for the $\ell^{1}$-norm of the gradient, is given by

$$
\forall u \in \mathbb{R}^{\Omega}, \forall x \in \Omega, \quad \partial T V(u)(x)=\sum_{y,|y-x|_{1}=1} \operatorname{sign}(u(x)-u(y)),
$$

and is hence included in $[-4,4]$. This implies that for each pixel $y \in \Omega$, the gray level $\hat{u}_{\delta}(y)$ satisfies

$$
\hat{u}_{\delta}(y) \in\left[v(y)+\delta \mathbb{1}_{\{x\}}(y)-2 \lambda, v(y)+\delta \mathbb{1}_{\{x\}}(y)+2 \lambda\right] .
$$

For $y=x$, we get

$$
\hat{u}_{\delta}(x) \in[v(x)+\delta-2 \lambda, v(x)+\delta+2 \lambda],
$$

which yields, when $\delta$ goes to $+\infty$,

$$
\frac{\hat{u}_{\delta}(x)-\hat{u}_{0}(x)}{\delta} \geq \frac{v(x)-\hat{u}_{0}(x)-2 \lambda}{\delta}+1 \underset{\delta \rightarrow+\infty}{\longrightarrow} 1,
$$

so that $C_{x}(x) \geq 1$. Now as $C_{x}(x) \leq 1$ (as shown above), we get the desired result.

(3) The TV-denoising operator $T$ is shown to be non-expansive (in [30, Lemma 2.4] for instance), that is

$$
\forall v_{1}, v_{2} \in \mathbb{R}^{\Omega}, \quad\left\|T\left(v_{2}\right)-T\left(v_{1}\right)\right\|_{2} \leq\left\|v_{2}-v_{1}\right\|_{2} .
$$

Applying this inequality to $v_{1}=v$ and $v_{2}=v+\delta \mathbb{1}_{\{x\}}$ yields

$$
\sum_{y \in \Omega}\left(\hat{u}_{\delta}(y)-\hat{u}_{0}(y)\right)^{2} \leq \delta^{2}
$$

which proves the desired result.

(2) As $T$ is a proximal operator associated to a convex function $(T V)$, it is maximal-monotone, which means that

$$
\forall v_{1}, v_{2} \in \mathbb{R}^{\Omega}, \quad\left\langle T\left(v_{2}\right)-T\left(v_{1}\right), v_{2}-v_{1}\right\rangle \geq 0,
$$

where $\langle f, g\rangle=\sum_{x \in \Omega} f(x) g(x)$ denotes the usual inner product. Let $b$ be an arbitrary real number, and set $v_{1}=v$ and $v_{2}=v+\delta \mathbb{1}_{\{x\}}+b$. The maximal-monotone property and the shift invariance of $T$ (11) yield

$$
\left\langle\hat{u}_{\delta}+b-\hat{u}_{0}, \delta \mathbb{1}_{\{x\}}+b\right\rangle \geq 0 .
$$

Expanding the inner product leads to

$$
\left\langle b \mathbb{1}_{\Omega}, \delta \mathbb{1}_{\{x\}}+b\right\rangle+\left\langle\hat{u}_{\delta}-\hat{u}_{0}, b \mathbb{1}_{\Omega}\right\rangle+\delta\left(\hat{u}_{\delta}(x)-\hat{u}_{0}(x)\right) \geq 0,
$$

and thanks to the average conservation property (10) of $T$ we get

$$
|\Omega| b^{2}+2 \delta b+\delta\left(\hat{u}_{\delta}(x)-\hat{u}_{0}(x)\right) \geq 0 .
$$


This inequality is true for any value of $b$, hence the discriminant of the quadratic polynomial is nonpositive, which leads, after simplifications, to

$$
\forall \delta \neq 0, \quad\left|\frac{\hat{u}_{\delta}(x)-\hat{u}_{0}(x)}{\delta}\right| \geq \frac{1}{|\Omega|} .
$$

Now using item (3) of the theorem, we can write for any $\delta \neq 0$

$$
\sum_{y \neq x}\left(\frac{\hat{u}_{\delta}(y)-\hat{u}_{0}(y)}{\delta}\right)^{2} \leq 1-\left(\frac{\hat{u}_{\delta}(x)-\hat{u}_{0}(x)}{\delta}\right)^{2} \leq 1-\frac{1}{|\Omega|^{2}} .
$$

This implies that for any $y \neq x$,

$$
C_{x}(y)^{2} \leq 1-\frac{1}{|\Omega|^{2}}
$$

which ends the proof.

\subsection{Experiments}

Using numerical experiments, we can investigate some properties of the influence map that are not considered in Theorem 1 . To compute $C_{x}$ numerically on 8-bits images, we use the approximation

$$
C_{x}(y) \simeq \max _{\delta \in\left\{ \pm 2^{n}, 0 \leq n \leq 9\right\}}\left|\frac{T\left(v+\delta \mathbb{1}_{\{x\}}\right)(y)-T(v)(y)}{\delta}\right|,
$$

where the TV-denoising operator $T$ is estimated with a very high precision using Chambolle's dual algorithm (the "max" version of [21], mentioned in [22]).

The first striking property of the influence map, shown on Figure 1, is the fast decrease of $C_{x}(y)$ as $y$ moves away from $x$. For typical values of $\lambda$ and most pixels $x, C_{x}(y)$ is below 0.01 as soon as $\|y-x\|$ is larger than 15 pixels. At this point, a natural question arises: is the support of an influence map much smaller than the image domain in general? Following the discussion just before Section 2.1, it is reasonable to think that the answer is no for Neumann boundary conditions (used in practice), in reason of the average-preserving property of TV-denoising in that case. However, in the case of an infinite domain or Dirichlet boundary conditions (Section 2.1), there are good reasons to think that in general, most (not to say all) influence maps have a small support. Since the exact support of $C_{x}$ is difficult to compute in numerical experiments, and in order to neglect the slight global effect resulting from the average preservation induced by Neumann boundary conditions, we propose to consider a significant support of $C_{x}$, defined by

$$
\operatorname{supp}_{\varepsilon}\left(C_{x}\right)=\left\{y \in \Omega, C_{x}(y)>\varepsilon\right\}
$$

where $\varepsilon$ is a small positive threshold ( $\varepsilon=0.01$ in practice). The boundary of this significant support is displayed on Figure 1 by the largest level line associated to each considered pixel $x$.

We computed the significant support of 100 random pixels of Lena image, and reported on Figure 2 the histograms of their associated radii (maximum distance from $x$ to a point of the significant support $\operatorname{supp}_{\varepsilon}\left(C_{x}\right)$ ), for three different values of the regularization parameter $\lambda$. As we can see, all significant supports are small, and they tend to grow when $\lambda$ increases (as for an averaging filter, whose smoothing effect increases with the size of the neighboring window).

In this part, we have brought theoretical and numerical evidence that TV-denoising is very near to be a local operator. In particular, we observed that for usual values of the denoising parameter $\lambda$, the influence of a given pixel was generally limited to a range of $10-15$ pixels. It could be interesting to further study the locality of TV-denoising, and in particular possibilities to compute exactly the true support of the influence map of a given pixel, for appropriate boundary conditions. However, since there 

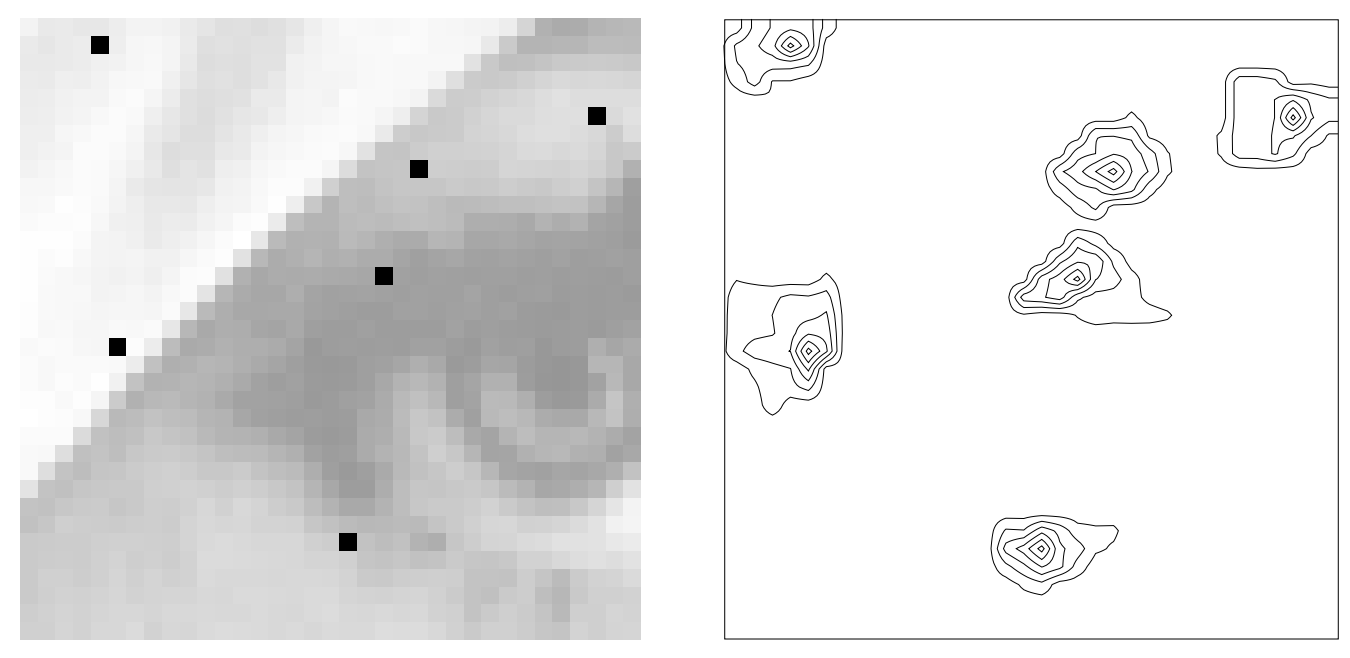

Figure 1: Six randomly chosen pixels of a part of Lena image (in black on the left image), and some level lines of their influence maps (right), corresponding to $\lambda=20$ and levels sampled between 0.01 and 1 on a regular logarithmic scale. As we can see, the influence map of each pixel is very concentrated, which suggests that TV-denoising is not far from being a local operator.
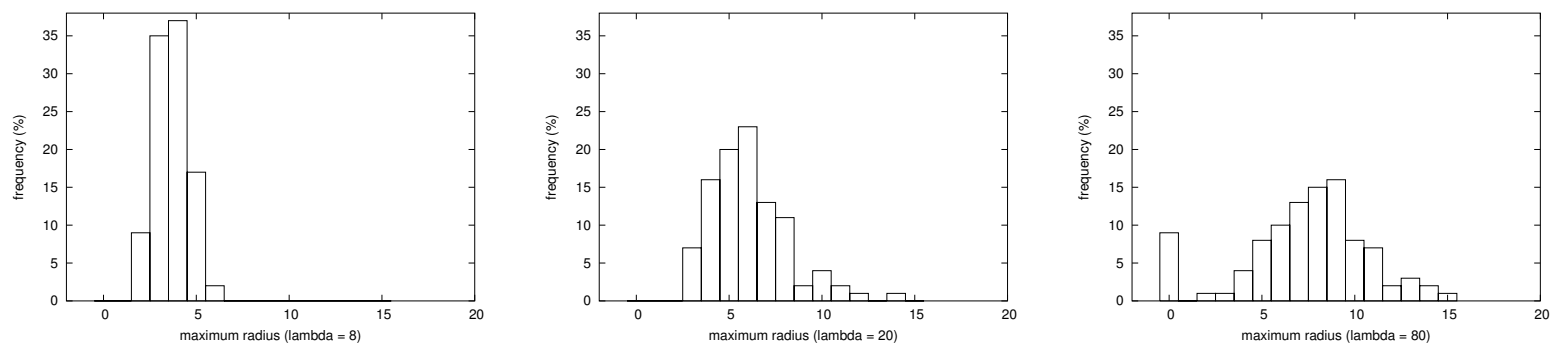

Figure 2: Given a (randomly chosen) pixel $x$, we numerically compute the significant support $\operatorname{supp}_{\varepsilon}$ $(\varepsilon=0.01)$ of the influence map $C_{x}$, and measure its radius, that is, the maximum Euclidean distance from $x$ to a point of $\operatorname{supp}_{\varepsilon}\left(C_{x}\right)$. This operation is performed on 100 random pixels of Lena image and yields a histogram of the 100 observed radii for 3 different values of the denoising level (left column: $\lambda=8$; middle column: $\lambda=20$; right column: $\lambda=80$ ). We can notice that as $\lambda$ increases, most pixels increase their influence zone (the significant support of $C_{x}$ gets larger), but the influence never propagate beyond 15 pixels. Moreover, for $\lambda=80$ (right column), $9 \%$ of the pixels have an influence map with a 1-pixel significant support (radius 0), which means that these pixels do not significantly influence other pixels than themselves. This phenomenon is likely to be a consequence of the staircasing effect, discussed in Section 6.3.

probably exists no uniform bound (with respect to pixels and images) on the maximal influence range, it becomes logical at this point, considering the discussion made in Introduction, to reformulate the original variational formulation of TV-denoising in the context of local filters, giving birth in the next section to what we call local $T V$-denoising. 


\section{Local TV-denoising}

\subsection{Motivation and definition}

In this section, we build a purely local filter inspired from TV-denoising: each pixel is processed using the pixels lying in its neighborhood. As we shall see, this construction opens interesting possibilities, such as deriving specific properties of local filters for an operator close to classical TV-denoising (Section 4), obtaining a TV-denoising method avoiding the "staircasing" artifact (Section 6), and prefiltering the patches of an image efficiently before a patch-based denoising method (Section 7).

Definition 1 We shall call neighborhood shape any finite subset $\mathcal{W}$ of $\mathbb{Z}^{2}$ that contains 0.

The most usual neighborhood shapes will be connected sets, and in particular rectangles or discrete balls, but it is interesting to notice that the construction we make here does not require any particular geometric assumption on the neighborhood used.

For any pixel $x \in \Omega$, we write $\mathcal{W}_{x}=(x+\mathcal{W}) \cap \Omega$ the neighborhood of $x$, and $v\left(\mathcal{W}_{x}\right) \in \mathbb{R}^{\mathcal{W}_{x}}$ the image $v$ restricted to this neighborhood. We propose to consider the denoising operator $T_{\lambda}^{\mathcal{W}}$ defined by

$$
\begin{gathered}
\forall v \in \mathbb{R}^{\Omega}, \forall x \in \Omega, \quad T_{\lambda}^{\mathcal{W}}(v)(x)=u_{x}(x) \\
\text { where for all } x \in \Omega, u_{x} \in \mathbb{R}^{\mathcal{W}_{x}} \text { minimizes }\left\|u_{x}-v\left(\mathcal{W}_{x}\right)\right\|^{2}+\lambda T V\left(u_{x}\right) .
\end{gathered}
$$

Of course here, if $w \in \mathbb{R}^{\mathcal{W}_{x}},\|w\|^{2}$ denotes the restricted squared $\ell^{2}$-norm $\sum_{y \in \mathcal{W}_{x}} w(y)^{2}$. Hence, $T_{\lambda}^{\mathcal{W}}$ is a local filter, as it amounts to minimizing a local ROF energy on every neighborhood $\mathcal{W}_{x}$ and keeping the central pixel value $u_{x}(x)$ only. It is also associated to TV-denoising because letting $\mathcal{W}=\mathbb{Z}^{2}$ leads to the classical global TV-denoising.

\subsection{Window weighting for artifact-free local TV-denoising}

Experiments carried out on local TV-denoising with large regularizing parameters $\lambda$ immediately reveal serious aliasing-like artifacts, that is, the artificial emergence of low frequencies. This phenomenon is particularly visible on signals (that can be considered as images made of a single row to extend previous notations). For instance on Figure 3, a chirp signal (left) is denoised using local TV-denoising and the result (middle) dramatically suffers from this artifact.

This aliasing-like artifact can be explained by the convergence of local TV-denoising towards linear filtering as the regularity parameter $\lambda$ tends to infinity. Indeed, as will be seen in Proposition 2 at the end of this section,

$$
T_{\lambda}^{\mathcal{W}}(v)(x) \underset{\lambda \rightarrow \infty}{\longrightarrow} \frac{1}{|\mathcal{W}|} \sum_{y \in \mathcal{W}_{x}} v(y),
$$

so that $T_{\mathcal{W}, \lambda}$ is asymptotically equivalent to a discrete convolution with a boxcar function, whose Fourier transform (cardinal sine function) is very oscillating. This explains the aliasing-like artifact for large $\lambda$ (we use the term "aliasing-like" because it is the interference between the boxcar fundamental frequency and the chirp local frequency that creates a low-frequency envelope).

By analogy with linear filtering, we propose to introduce weights on the window $\mathcal{W}$ to attenuate this artifact.

Definition 2 (local weighted TV-denoising) Let $\omega=\left(\omega_{x}\right) \in \mathbb{R}^{\mathcal{W}}$ be positive weights associated to the neighborhood shape $\mathcal{W}$, and let $\|\cdot\|_{\omega}$ denote the weighted Euclidean norm defined by

$$
\forall u \in \mathbb{R}^{\mathcal{W}_{x}}, \quad\|u\|_{\omega}^{2}=\sum_{y \in \mathcal{W}_{x}} \omega_{y-x} u(y)^{2} .
$$



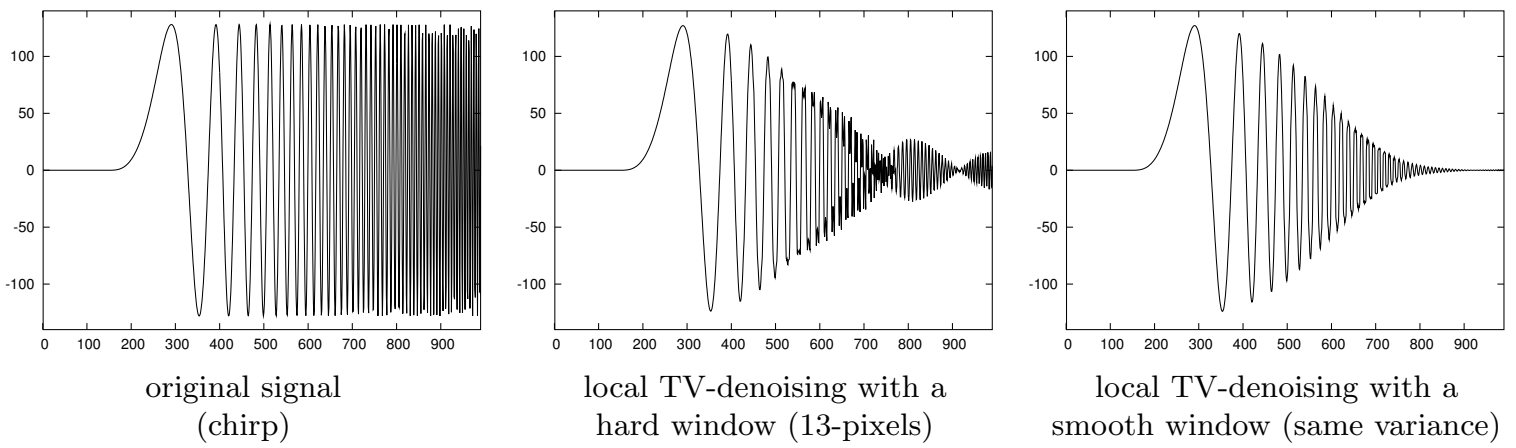

local TV-denoising with a smooth window (same variance)

Figure 3: Effect of local TV-denoising on the chirp. A chirp signal (left) is denoised with the local TV-filter associated to a neighborhood of size 13 (discrete interval), with $\lambda=500$ (middle). Contrary to what could be legitimately expected, frequencies are not attenuated in a monotone way, and low-frequency "waves" (interferences) can be observed on the right part of the signal. This aliasing-like phenomenon, that would appear in a similar way with a boxcar convolution, can be strongly reduced by using an appropriate weight function in the fidelity term ( $L^{2}$ norm) of the variational formulation. This improvement can be observed on the right, where the weighted local TV-denoising introduced in this section has been applied to the chirp signal with a Gaussian weight function of variance $a^{2}=14$ (same variance than the hard window) truncated to a large enough window (21 pixels).

Then, the local weighted $T V$-denoiser $T_{\omega, \lambda}$ is an operator on $\mathbb{R}^{\Omega}$ defined by

$$
\begin{gathered}
\forall v \in \mathbb{R}^{\Omega}, \forall x \in \Omega, \quad T_{\omega, \lambda}(v)(x)=u_{x}(x) \\
\text { where } u_{x} \in \mathbb{R}^{\mathcal{W}_{x}} \text { minimizes } E_{\lambda, \omega, x}\left(u_{x}\right)=\left\|u_{x}-v\left(\mathcal{W}_{x}\right)\right\|_{\omega}^{2}+\lambda T V\left(u_{x}\right) .
\end{gathered}
$$

Proposition 2 below gives the asymptotic behavior of the local weighted TV-denoiser when $\lambda$ goes to $+\infty$.

Proposition 2 For any positive weight function $\omega \in \mathbb{R}^{\mathcal{W}}$, one has

$$
\forall v \in \mathbb{R}^{\Omega}, \forall x \in \Omega, \quad T_{\omega, \lambda}(v)(x) \underset{\lambda \rightarrow+\infty}{\longrightarrow} \frac{\sum_{y \in \mathcal{W}_{x}} \omega_{y-x} v(y)}{\sum_{y \in \mathcal{W}_{x}} \omega_{y-x}} .
$$

Proof $-\mathrm{A}$ point $x \in \Omega$ being fixed, we write $\bar{u}$ the local weighted average around $x$ of an image $u$, that is,

$$
\forall u \in \mathbb{R}^{\mathcal{W}_{x}}, \quad \bar{u}=\frac{\sum_{y \in \mathcal{W}_{x}} \omega_{y-x} u(y)}{\sum_{y \in \mathcal{W}_{x}} \omega_{y-x}} .
$$

If $u_{\lambda}$ is the minimizer of $E_{\lambda, \omega, x}$, then 0 is the minimizer of $J: \mathbb{R} \rightarrow \mathbb{R}$ defined by

$$
J(t)=E_{\lambda, \omega, x}\left(u_{\lambda}+t \mathbb{1}\right)=\left\|u_{\lambda}+t \mathbb{1}-v\left(\mathcal{W}_{x}\right)\right\|_{\omega}^{2}+\lambda T V\left(u_{\lambda}\right),
$$

(where $\mathbb{1}$ denotes the constant image equal to 1 everywhere), and writing $J^{\prime}(0)=0$ immediately leads to $\bar{u}_{\lambda}=\bar{v}$. Now, as the norms $u \mapsto\|u\|_{\infty}$ and $u \mapsto T V(u)+|\bar{u}|$ are equivalent in the finite dimensional space $\mathbb{R}^{\mathcal{W}_{x}}$, there exists a constant $C>0$ such that

$$
\forall u \in \mathbb{R}^{\mathcal{W}_{x}}, \quad C\|u\|_{\infty} \leq T V(u)+|\bar{u}| .
$$

Applying this inequality to $u_{\lambda}-\bar{u}_{\lambda}=u_{\lambda}-\bar{v}$, we get

$$
C\left\|u_{\lambda}-\bar{v}\right\|_{\infty} \leq T V\left(u_{\lambda}-\bar{v}\right)=T V\left(u_{\lambda}\right) \leq \frac{1}{\lambda} E_{\lambda, \omega, x}\left(u_{\lambda}\right) \leq \frac{1}{\lambda} E_{\lambda, \omega, x}(\bar{v})=\frac{1}{\lambda}\|v-\bar{v}\|_{\omega}^{2} \underset{\lambda \rightarrow+\infty}{\longrightarrow} 0,
$$


so that in particular $T_{\omega, \lambda}(v)(x)=u_{\lambda}(x) \underset{\lambda \rightarrow+\infty}{\longrightarrow} \bar{v}$.

Proposition 2 states that $T_{\omega, \lambda}$ is asymptotically equivalent (when $\lambda$ goes to $+\infty$ ) to a linear convolution by the finitely supported kernel $\tilde{\omega}: x \mapsto \omega_{-x}$. Another viewpoint is to let the global contrast of the image go to 0 (which is equivalent to the case $\lambda \rightarrow+\infty$ thanks to the change of variable $v \mapsto \alpha v$ ), and this leads, for a fixed $\lambda$, to

$$
T_{\omega, \lambda}(\alpha v)(x) \underset{\alpha \rightarrow 0}{\sim} \alpha \frac{\sum_{y \in \mathcal{W}_{x}} \omega_{y-x} v(y)}{\sum_{y \in \mathcal{W}_{x}} \omega_{y-x}} .
$$

Hence, we expect the local TV-denoising to behave like a linear filter in low-contrasted regions or when $\lambda$ is large (indeed, we shall see later in Section 4.4.1 that this linear regime is actually attained for a finite $\lambda)$.

Now, coming back to the aliasing-like artifacts noticed before, it is well known that a linear convolution will avoid aliasing-like artifacts for smooth kernels that have an non-oscillating (unimodal) Fourier Transform, and in particular for Gaussian weights defined by

$$
\forall x \in \mathcal{W}, \quad \omega_{x}=\exp \left(-\frac{|x|^{2}}{2 a^{2}}\right) \quad(\text { with } a>0) .
$$

Figure 3 (right) shows the result obtained after applying local weighted TV-denoising (with the Gaussian weights given by Equation 28) on the chirp signal, with an algorithm that will be detailed in Section 5 . As expected, the aliasing-like artifacts are removed.

\section{Properties}

In this section, we investigate several properties of the above-proposed local TV-denoising filter, in particular local and global comparison principles and asymptotic behaviors.

\subsection{Local comparison}

An important interest of having a local filter instead of a global minimization process is to be able to control the denoising process more locally and more accurately. Among interesting properties are the local comparison principle, that offers a guarantee of local stability. This principle does not always hold for global TV-denoising, but the following proposition states that it does for local TV-denoising (weighted or unweighted).

Proposition 3 (Local comparison principle) Let $T V$ be defined in (3) either with a $\ell^{1}$ or a $\ell^{2}$ norm of the gradient (Equation 6 or 9). Then the local filter $T_{\omega, \lambda}$ applied on an image $v$ satisfies

$$
\forall x \in \Omega, \quad \min _{y \in \mathcal{W}_{x}} v(y) \leq T_{\omega, \lambda} v(x) \leq \max _{y \in \mathcal{W}_{x}} v(y) .
$$

The value of the denoised image hence lies in the local dynamic range of the noisy image. This stability property also echoes the fact that the local TV-denoiser has a more limited denoising level (when $\lambda \rightarrow+\infty$, see Equation 25) than global TV-denoising (for which the most denoised image is a global constant).

Proof -Assume that there exists $x \in \Omega$ such that $T_{\omega, \lambda}(v)(x) \notin[m, M]$, where $m=\min _{y \in \mathcal{W}_{x}} v(y)$ and $M=\max _{y \in \mathcal{W}_{x}} v(y)$. Let $u_{x} \in \mathbb{R}^{\mathcal{W}_{x}}$ be the patch associated to the denoising of $v\left(\mathcal{W}_{x}\right)$ i.e. minimizing (24). Let now $u_{x}^{\prime}$ be the patch with saturated values defined by

$$
\forall y \in \mathcal{W}_{x}, \quad u_{x}^{\prime}(y)=\left\{\begin{array}{lll}
m & \text { if } & u_{x}(y) \leq m, \\
u_{x}(y) & \text { if } & m \leq u_{x}(y) \leq M, \\
M & \text { if } & M \leq u_{x}(y)
\end{array}\right.
$$


Then $\left\|u_{x}^{\prime}-v\left(\mathcal{W}_{x}\right)\right\|_{\omega}^{2}<\left\|u_{x}-v\left(\mathcal{W}_{x}\right)\right\|_{\omega}^{2}$ since the gray levels $u_{x}(y)$ lying away from $[m, M]$ are changed into values which are closer to the noised value $v(y)$. Besides, as

$$
\forall y, z \in \mathcal{W}_{x}, \quad\left|u_{x}^{\prime}(z)-u_{x}^{\prime}(y)\right| \leq\left|u_{x}(z)-u_{x}(y)\right|,
$$

(because $u_{x} \mapsto u_{x}^{\prime}$ is 1-Lipschitz), we have $T V\left(u_{x}^{\prime}\right) \leq T V\left(u_{x}\right)$. This implies that $E_{\lambda, \omega, x}\left(u_{x}^{\prime}\right)<E_{\lambda, \omega, x}\left(u_{x}\right)$, which contradicts the minimality of $E_{\lambda, \omega, x}\left(u_{x}\right)$.

\subsection{Monotony}

A global comparison principle that is desirable for general image denoising is the following: if two noisy images $v_{1}$ and $v_{2}$ satisfy the inequality $v_{1}<v_{2}$ (pointwise), then it would be expected that the related denoised images satisfy a similar large inequality $T\left(v_{1}\right) \leq T\left(v_{2}\right)$. This so-called monotony property is an interesting property, since it extends the stability behavior from smooth (say, Lipschitz or more) to non-smooth images, as a non-smooth image can always be bounded from below and above by two smooth images.

A convolution filter is monotone as soon as its convolution kernel is non-negative. Local filters such as the bilateral filter or Non-Local means, according to our experiments, are generally not monotone (some asymptotic behaviors can be monotone, though). Now for global TV-denoising, it has been shown that the monotony property was true in the continuous framework $[3,4,18]$, and in the discrete framework with a $\ell^{1}$ scheme for the gradient norm $[22,34]$. However it seems that the monotony does not hold any more in the discrete framework with a $\ell^{2}$ scheme for the gradient norm (Equation 9). This can be seen on the following numerical example made of $3 \times 3$-pixel images: if we take

$$
v_{1}=\begin{array}{|c|c|c|}
\hline 42 & 94 & 254 \\
\hline 76 & 178 & 18 \\
\hline 0 & 0 & 0 \\
\hline
\end{array} \quad \text { and } \quad v_{2}=\begin{array}{|c|c|c|c|}
\hline 43 & 95 & 255 \\
\hline 77 & 179 & 19 \\
\hline 60 & 69 & 105 \\
\hline
\end{array}
$$

then after a global TV-denoising with a $\ell^{2}$ scheme $(\lambda=30)$ we obtain

$$
T\left(v_{1}\right) \approx \begin{array}{|c|c|c|}
\hline 60.81 & 98.68 & 224.78 \\
\hline 72.73 & 140.87 & 27.89 \\
\hline 12.08 & 12.08 & 12.08 \\
\hline
\end{array} \quad \text { and } \quad T\left(v_{2}\right) \approx \begin{array}{|c|c|c|c|}
\hline 63.29 & 100.49 & 225.65 \\
\hline 83.12 & 138.65 & 60.74 \\
\hline 76.69 & 76.69 & 76.69 \\
\hline
\end{array} \text {. }
$$

Hence we have $v_{1}<v_{2}$ but $T\left(v_{1}\right) \not \leq T\left(v_{2}\right)$ (because of the central pixel). This counter-example still holds when the same little images are included in larger constant images set to 0 .

Unweighted local TV-denoising directly inherits the possible monotony property of global TV-denoising, as next proposition states.

Proposition 4 (Global comparison principle) Assume that the global TV-denoising is monotone. Then the local $T V$-denoising is monotone. In particular, local $T V$-denoising defined with a $\ell^{1}$ scheme for the gradient norm (Equation 6) is monotone.

Proof - Let $v_{1}<v_{2}$ and let $x \in \Omega$. Let $p_{1}$ and $p_{2}$ denote the patches which minimize the local ROF energies associated to $v_{1}\left(\mathcal{W}_{x}\right)$ and $v_{2}\left(\mathcal{W}_{x}\right)$ respectively. The monotony property of the global TVdenoising operator on $v_{1}\left(\mathcal{W}_{x}\right)<v_{2}\left(\mathcal{W}_{x}\right)$ implies that $p_{1} \leq p_{2}$. Hence, denoting $p_{i}(0)$ the central gray level of the patch $p_{i}$, we get $T_{\mathcal{W}, \lambda} v_{1}(x)=p_{1}(0) \leq p_{2}(0)=T_{\mathcal{W}, \lambda} v_{2}(x)$.

The proposed local TV-denoising essentially depends on two parameters : the regularization coefficient $\lambda$ and the weights $\left(\omega_{y}\right)$ which in particular control the locality. As we shall see in the following sections, $\omega$ and $\lambda$ behave in opposite directions. More specifically, when $\lambda \rightarrow 0$, the denoising becomes equivalent to the global denoising, and when the neighborhood becomes infinitely small, the denoising becomes equivalent to a linear filtering, as if $\lambda \rightarrow+\infty$. 


\subsection{Asymptotics for $\lambda \rightarrow 0$}

\subsubsection{Asymptotic equivalence to global TV-denoising when $\lambda \rightarrow 0$}

In this section we show that local and global TV-denoising have the same asymptotic behavior when $\lambda \rightarrow 0$.

Theorem 2 Let $\mathcal{W}$ be a neighborhood shape, and $\omega \in \mathbb{R}^{\mathcal{W}}$ a positive weight function. For any image $v \in \mathbb{R}^{\Omega}$ and any $x \in \Omega$ such that $\mathcal{W}_{x}$ contains $x$ and its 8 nearest neighbors, and such that $T V$ is continuously differentiable in the neighborhood of $v\left(\mathcal{W}_{x}\right)$, we have

$$
T_{\omega, \lambda} v(x)=v(x)-\frac{\lambda}{2 \omega_{0}} \nabla T V(v)(x)+\underset{\lambda \rightarrow 0}{o}(\lambda) .
$$

Proof $-T_{\omega, \lambda} v(x)$ is the central gray level of the patch $u \in \mathbb{R}^{\mathcal{W}_{x}}$ which minimizes the energy $E_{\lambda, \omega, x}$ in (24), and whose subdifferential then satisfies

$$
\forall y \in \mathcal{W}_{x}, \quad 2 \omega_{y-x}(u(y)-v(y))+\lambda \partial T V(u)(y) \ni 0 .
$$

Let us introduce

$$
w_{\lambda}=\frac{u-v\left(\mathcal{W}_{x}\right)}{\lambda}
$$

Since $E_{\lambda, \omega, x}\left(v\left(\mathcal{W}_{x}\right)\right)=\lambda T V\left(v\left(\mathcal{W}_{x}\right)\right) \rightarrow 0$ when $\lambda \rightarrow 0$, we have $\left\|u-v\left(\mathcal{W}_{x}\right)\right\|_{\omega}^{2} \rightarrow 0$ and thus $\lambda w_{\lambda} \rightarrow 0$ since the weight function $\omega$ is positive. Now (30) writes, for $y=x$,

$$
2 \omega_{0} w_{\lambda}(x)+\partial T V\left(v\left(\mathcal{W}_{x}\right)+\lambda w_{\lambda}\right)(x) \ni 0 .
$$

As $T V$ is assumed to be continuously differentiable at the neighborhood of $v\left(\mathcal{W}_{x}\right)$, when $\lambda$ is small enough, $\partial T V\left(v\left(\mathcal{W}_{x}\right)+\lambda w_{\lambda}\right)(x)$ is equal to $\left\{\nabla T V\left(v\left(\mathcal{W}_{x}\right)+\lambda w_{\lambda}\right)(x)\right\}$, and

$$
\nabla T V\left(v\left(\mathcal{W}_{x}\right)+\lambda w_{\lambda}\right)(x) \underset{\lambda \rightarrow 0}{\longrightarrow} \nabla T V\left(v\left(\mathcal{W}_{x}\right)\right)(x)=\nabla T V(v)(x)
$$

since the window $\mathcal{W}_{x}$ contains $x$ and its 8 nearest neighbors (actually 6 neighbors are enough). Finally,

$$
w_{\lambda}(x)=-\frac{1}{2 \omega_{0}} \nabla T V(v)(x)+\underset{\lambda \rightarrow 0}{o}(1),
$$

so that $u(x)=v(x)-\frac{\lambda}{2 \omega_{0}} \nabla T V(v)(x)+\underset{\lambda \rightarrow 0}{o}(\lambda)$ and the theorem is proven.

Remark: In Theorem 2, $T V$ needs to be continuously differentiable in the neighborhood of $v\left(\mathcal{W}_{x}\right)$. As $T V$ is smooth almost everywhere, this is not a strong assumption in practice, since this is true for almost every noisy image $v$ (provided that the noise process admits a density with respect to Lebesgue measure).

In a continuous framework, $-\nabla T V$ corresponds to the curvature operator

$$
\operatorname{curv}(u)=\operatorname{div}\left(\frac{\nabla u}{|\nabla u|}\right)
$$

and in this case the result of Theorem 2 would be

$$
T_{\omega, \lambda} v(x)=v(x)+\frac{\lambda}{2 \omega_{0}} \operatorname{curv}(v)(x)+\underset{\lambda \rightarrow 0}{o}(\lambda) .
$$

This implies that the limiting partial differential equation (PDE) associated to iterated $T_{\omega, \lambda}$ is

$$
\frac{\partial u}{\partial t}=\operatorname{curv} u
$$

which is the PDE associated to global TV-denoising $[6,55]$, that also corresponds to a degenerate case of Perona-Malik Equation [52]. 


\subsubsection{Application to the normalization of weights}

If we want to compare the respective influence of two weight functions $\omega$ and $\omega^{\prime}$ for a fixed value of the parameter $\lambda$, we need to impose a normalization procedure for the weight functions. In particular, all weight functions $\alpha \omega$ (for $\alpha>0$ ) must be normalized into the same weight function, since the local TVdenoising obtained with $(\omega, \lambda)$ and $(\alpha \omega, \lambda / \alpha)$ are exactly the same. Several constraint equations could be used for weight normalization, for example $\sum_{y} \omega_{y}=1$, or $\sum_{y} \omega_{y}^{2}=1$, or $\frac{1}{|\mathcal{W}|} \sum_{y} \omega_{y}=1$, or $\max _{y} \omega_{y}=1$, etc. A particular normalization is suggested by Equation (29). Indeed, since the asymptotic behavior of the local TV-denoising of a given patch only depends (at first order) on $\lambda / \omega_{0}$ and not on the other weight coefficients $\left(\omega_{y}\right)_{y \neq 0}$, it is natural to impose a fixed value of $\omega_{0}$ as a weight normalization, so that the asymptotic behavior of local TV-denoising only depends on $\lambda$ (as it is the case for global TV-denoising). Hence, in the following all weight functions will be normalized according to the constraint $\omega_{0}=1$, as was done in (28).

\subsection{Asymptotics for $\lambda \rightarrow+\infty$}

\subsubsection{Linear regime reached for finite $\lambda$}

Here we extend Proposition 2 by showing that the limiting linear regime is reached for a finite $\lambda$. This can be seen as the finite counterpart of [20, Lemma 2.3] with an elementary proof.

Proposition 5 Let $\mathcal{W}$ be a neighborhood shape and $\omega \in \mathbb{R}^{\mathcal{W}}$ a positive weight function. For any image $v \in \mathbb{R}^{\Omega}$ and any $x \in \Omega$, there exists a critical value denoted by $\lambda_{c}(x)$ such that

$$
\forall \lambda \geq \lambda_{c}(x), \quad T_{\omega, \lambda}(v)(x)=\frac{\sum_{y \in \mathcal{W}_{x}} \omega_{y-x} v(y)}{\sum_{y \in \mathcal{W}_{x}} \omega_{y-x}}
$$

Proof - Let $\bar{v}=\frac{\sum_{y \in \mathcal{W}_{x}} \omega_{y-x} v(y)}{\sum_{y \in \mathcal{W}_{x}} \omega_{y-x}}$. We prove that the constant image $\bar{v}$ achieves the minimum of $E_{\lambda, \omega, x}$ (defined in (24)) by considering the behavior of $E_{\lambda, \omega, x}$ at the neighborhood of $\bar{v}$. Let $u \in \mathbb{R}^{\mathcal{W}_{x}}$; it can be decomposed into $u=\delta u+\bar{v}+\alpha$ where $\delta u$ is an image with zero weighted mean, $\alpha$ is a scalar.

First, denoting $\langle\cdot, \cdot\rangle_{\omega}$ the inner product associated to the Hilbert norm $\|\cdot\|_{\omega}$, and $\mathbb{1}$ the constant image equal to 1 everywhere on $\mathcal{N}_{x}$, notice that

$$
E_{\lambda, \omega, x}(\delta u+\bar{v}+\alpha)-E_{\lambda, \omega, x}(\delta u+\bar{v})=\|\alpha \mathbb{1}\|_{\omega}^{2}+2\langle\alpha \mathbb{1}, \delta u+\bar{v}-v\rangle_{\omega}=\|\alpha \mathbb{1}\|_{\omega}^{2}
$$

since $\delta u+\bar{v}-v$ has zero weighted mean. Secondly,

$$
E_{\lambda, \omega, x}(\delta u+\bar{v})-E_{\lambda, \omega, x}(\bar{v} \mathbb{1})=\|\delta u\|_{\omega}^{2}+2\langle\bar{v}-v, \delta u\rangle_{\omega}+\lambda T V(\delta u),
$$

and setting $m(w)=\frac{1}{|\mathcal{W}|} \sum_{x \in \mathcal{W}} w(x)$ yields

$$
2\langle\bar{v}-v, \delta u\rangle_{\omega}=2\langle\bar{v}-v, \delta u-m(\delta u)\rangle_{\omega} \geq-2\|\omega(\cdot-x)(v-\bar{v})\|_{\infty}\|\delta u-m(\delta u)\|_{1} .
$$

Now, as all the norms are equivalent in finite dimension, there exists $C>0$ such that

$$
\forall w \in \mathbb{R}^{\mathcal{W}_{x}}, \quad|m(w)|+T V(w) \geq C\|w\|_{1} .
$$

Applying this inequality to $w=\delta u-m(\delta u)$, we get $T V(\delta u) \geq C\|\delta u-m(\delta u)\|_{1}$, so that with (32) and (33) we obtain

$$
E_{\lambda, \omega, x}(\bar{v}+\delta u)-E_{\lambda, \omega, x}(\bar{v} \mathbb{1}) \geq\|\delta u\|_{\omega}^{2}+\left(C \lambda-2\|\omega(\cdot-x)(v-\bar{v})\|_{\infty}\right)\|\delta u-m(\delta u)\|_{1} .
$$


Last, adding (31) to (35) yields

$$
E_{\lambda, \omega, x}(u)-E_{\lambda, \omega, x}(\bar{v} \mathbb{1}) \geq\|\alpha \mathbb{1}\|_{\omega}^{2}+\|\delta u\|_{\omega}^{2}+\left(C \lambda-2\|\omega(\cdot-x)(v-\bar{v})\|_{\infty}\right)\|\delta u-m(\delta u)\|_{1}
$$

and the right-hand term is nonnegative for any $u \in \mathbb{R}^{\mathcal{W}}$ as soon as $\lambda \geq 2\|\omega(\cdot-x)(v-\bar{v})\|_{\infty} / C$. It means that for large enough values of $\lambda, \bar{v} \mathbb{1}$ reaches the minimum of $E_{\lambda, \omega, x}$.

\subsubsection{Asymptotic behavior for small neighborhoods}

Here we show that in a continuous setting, letting the size of the neighborhood $\mathcal{W}$ go to 0 (while keeping $\lambda$ constant) is equivalent to letting $\lambda \rightarrow+\infty$, hence asymptotically leading to linear filtering.

We first define local TV-denoising in a continuous setting. Let $\Omega$ denote an open subset of $\mathbb{R}^{2}$, and let $\mathcal{W}$ be a bounded convex open subset of $\mathbb{R}^{2}$ containing 0 , weighted by a positive function $\omega \in L^{\infty}(\mathcal{W})$. If $x \in \Omega$, the set $\mathcal{W}_{x}=(x+\mathcal{W}) \cap \Omega$ is a neighborhood of $x$. The total variation of a patch $u \in L^{2}\left(\mathcal{W}_{x}\right)$ is defined by duality by

$$
T V(u)=\sup \left\{\int_{\mathcal{W}_{x}} u \operatorname{div} p, p \in \mathcal{C}_{c}^{\infty}\left(\mathcal{W}_{x}, \mathbb{R}^{2}\right),\|p\|_{\infty} \leq 1\right\}
$$

(see [7]), and its weighted norm $\|u\|_{\omega}$ with

$$
\|u\|_{\omega}^{2}=\int_{\mathcal{W}_{x}} \omega(y-x) u(y)^{2} d y
$$

Given $\mathcal{W}$ and $\lambda>0$, we consider the unique function $u$ belonging to

$$
B V\left(\mathcal{W}_{x}\right)=\left\{u \in L^{2}\left(\mathcal{W}_{x}\right), T V(u)<+\infty\right\}
$$

that minimizes

$$
E_{\lambda, \omega, x}(u)=\left\|u-v\left(\mathcal{W}_{x}\right)\right\|_{\omega}^{2}+\lambda T V(u),
$$

where $v\left(\mathcal{W}_{x}\right)$ denotes the restriction of $v$ on the subdomain $\mathcal{W}_{x}$. Then, the local TV-denoising operator $T_{\mathcal{W}, \lambda}$ at point $x$ is defined by

$$
T_{\mathcal{W}, \lambda} v(x)=\lim _{r \rightarrow 0^{+}} \frac{1}{|B(x, r)|} \int_{B(x, r)} u
$$

when the limit exists $(B(x, r)$ is the Euclidean open ball with center $x$ and radius $r$, and $|B(x, r)|$ its Lebesgue measure).

Note that in (39), we need to consider a limit (the mean value of $u$ at point $x$ ) because the value $u(x)$ has no meaning by itself ( $u$ is defined up to a Lebesgue-negligible function). Since $u \in B V\left(\mathcal{W}_{x}\right)$, this limit exists almost surely (that is, for almost any point of $\mathcal{W}_{x}$ ), which does not prove that $T_{\mathcal{W}, \lambda}$ is defined almost everywhere though (even if we believe that it is the case). In the following, the existence of $T_{\mathcal{W}, \lambda} v(x)$ will always be ensured in the asymptotic frameworks we consider.

Now, in order to make the neighborhood $\mathcal{W}$ shrink to a singleton, we consider a dilation parameter $h>$ 0 and the dilated set $h \mathcal{W}$, associated to the weight function $\omega(\cdot / h)$, in agreement with the normalization suggested in Section 4.3.2. The following theorem describes the asymptotic behavior of $T_{h \mathcal{W}, \lambda}$ when $h$ goes to 0 .

Theorem 3 Let $\Omega$ be an open subset of $\mathbb{R}^{2}$ and $v \in \mathcal{C}^{3}(\Omega)$. Let $\mathcal{W}$ a bounded convex open neighborhood of 0 in $\mathbb{R}^{2}$ and $\omega \in L^{\infty}(\mathcal{W})$ satisfying

$$
\omega>0, \quad \int_{\mathcal{W}} \omega(x) x d x=0, \quad \text { and }
$$




$$
\operatorname{Cov}_{\mathcal{W}}(\omega):=\frac{1}{\int_{\mathcal{W}} \omega}\left(\begin{array}{cc}
\int_{\mathcal{W}} x_{1}^{2} \omega(x) d x & \int_{\mathcal{W}} x_{1} x_{2} \omega(x) d x \\
\int_{\mathcal{W}} x_{1} x_{2} \omega(x) d x & \int_{\mathcal{W}} x_{2}^{2} \omega(x) d x
\end{array}\right)=\sigma^{2} I d
$$

(with the usual convention $x=\left(x_{1}, x_{2}\right)$ ). For each $x \in \Omega$, when $h$ is small enough, the denoising operator $T_{h \mathcal{W}, \lambda}$ considered by (39) is well defined and satisfies

$$
T_{h \mathcal{W}, \lambda} v(x)=v(x)+\frac{h^{2} \sigma^{2}}{2} \Delta v(x)+\underset{h \rightarrow 0}{O}\left(h^{3}\right),
$$

where $\Delta v=\frac{\partial^{2} v}{\partial x_{1}^{2}}+\frac{\partial^{2} v}{\partial x_{2}^{2}}$ denotes the Laplacian of $v$.

It is quite surprising that a restoration method based on Total Variation, which assigns a finite cost to contrasted edges but favors piecewise constant structures more than smooth structures (staircasing effect), could be associated to an isotropic diffusion through the Laplacian operator. Indeed, local TV-denoising is equivalent to global TV-denoising for large enough neighborhoods (see Section 6.1), but Theorem 3 above points out here that it is equivalent to Gaussian filtering for very small neighborhoods. This can be linked to the linear behavior of local TV-denoising in low-contrasted regions (Equation 27). Local TV-denoising with middle-sized neighborhoods hence reaches a compromise between global TV-denoising and Gaussian filtering.

Lemma 1 Let $v \in L^{2}(\Omega)$ and $x \in \Omega$. Assume that $\mathcal{W}$ is an open subset of $\mathbb{R}^{2}$ such that $\mathcal{W}_{x}$ is bounded and convex, and consider a positive weight function $\omega \in L^{\infty}(\mathcal{W})$. Let $\bar{v}_{x}=\frac{\int_{\mathcal{W}_{x}} \omega(y-x) v(y) d y}{\int_{\mathcal{W}_{x}} \omega(y-x) d y}$. If

$$
\lambda \geq \operatorname{diam}\left(\mathcal{W}_{x}\right) \cdot\left\|\omega(\cdot-x)\left(v\left(\mathcal{W}_{x}\right)-\bar{v}_{x}\right)\right\|_{L^{\infty}\left(\mathcal{W}_{x}\right)}
$$

$\left(\right.$ where $\operatorname{diam}\left(\mathcal{W}_{x}\right)$ denotes the diameter of $\left.\mathcal{W}_{x}\right)$, then the constant image $\bar{v}_{x}$ minimizes $E_{\lambda, \omega, x}$.

Remark : The existence of such a bound on $\lambda$ (Equation 41) above which the constant image minimizes $E_{\lambda, \omega, x}$ is proven in [20, Lemma 2.3] in a slightly different framework (the authors consider the eventuality of a blurring operator, but $\omega=1$ ). Here we derive an explicit upper bound and propose a proof directly inspired from the discrete framework.

Proof of Lemma 1 - The proof is similar to that of the discrete framework in Proposition 5. Only (34) has to be justified, with $C=2 / \operatorname{diam}\left(\mathcal{W}_{x}\right)$. Actually, in a continuous framework, the Poincaré inequality [7] states that for some constant $\gamma>0$, one has

$$
\forall u \in L^{1}\left(\mathcal{W}_{x}\right), \quad\|u-m(u)\|_{L^{1}\left(\mathcal{W}_{x}\right)} \leq \gamma T V(u)
$$

where $m(u)=\frac{1}{|\mathcal{W}|} \int_{\mathcal{W}} u(y) d y$. In [1], it is shown that if $\mathcal{W}_{x}$ is bounded and convex, the previous inequality holds with $\gamma=\operatorname{diam}\left(\mathcal{W}_{x}\right) / 2=1 / C$, which completes the proof.

Proof of Theorem 3 - For any $h>0$ and $x \in \Omega$, let $\mathcal{W}_{h, x}=(x+h \mathcal{W}) \cap \Omega$ and

$$
\bar{v}_{h, x}=\frac{\int_{\mathcal{W}} \omega(y) v(x+h t) d t}{\int_{\mathcal{W}} \omega(t) d t} .
$$

Consider $h_{0}>0$ such that for any $h<h_{0}, \mathcal{W}_{h, x} \subset \Omega$ (and hence is convex). By Lemma 1,

$$
\lambda_{h, x}^{c}=\operatorname{diam}\left(\mathcal{W}_{h, x}\right) \cdot\left\|\omega((\cdot-x) / h)\left(v\left(\mathcal{W}_{h, x}\right)-\bar{v}_{h, x}\right)\right\|_{L^{\infty}\left(\mathcal{W}_{h, x}\right)}
$$

is a critical value of $\lambda$, that is a value above which the denoised version of $v\left(\mathcal{W}_{h, x}\right)$ is constant, equal to $\bar{v}_{h, x}$. Now we have

$$
\lambda_{h, x}^{c} \leq h \operatorname{diam}(\mathcal{W}) \cdot 2\|\omega\|_{L^{\infty}(\mathcal{W})}\left\|v\left(\mathcal{W}_{x}\right)\right\|_{L^{\infty}\left(\mathcal{W}_{h_{0}, x}\right) \underset{h \rightarrow 0}{\longrightarrow} 0}
$$


hence there exists $h_{1} \in\left(0, h_{0}\right]$ such that $\lambda \geq \lambda_{h, x}^{c}$ as soon as $h<h_{1}$. Thus, for any $h<h_{1}$, the denoising is linear at point $x$, and $T_{h \mathcal{W}, \lambda} v(x)=\bar{v}_{h, x}$.

We end the proof by deriving an asymptotic development of $\bar{v}_{h, x}$ when $h \rightarrow 0$. As $v$ is $\mathcal{C}^{3}$, we can write for any $h \in\left(0, h_{1}\right)$,

$$
\forall t \in \mathcal{W}, \quad-M h^{3} \leq v(x+h t)-v(x)-h \nabla v(x) \cdot t-\frac{h^{2}}{2} D^{2} v(x)(t, t) \leq M h^{3},
$$

where $M=\frac{1}{6} \cdot \sup _{h_{1} \mathcal{W}}\left\|D^{3} v\right\| \cdot \sup _{h_{1} \mathcal{W}}|t|^{3}$ (notice that $M<+\infty$ because $D^{3} v$ is continuous on the closed set $\left.h_{1} \overline{\mathcal{W}}\right)$. Multiplying all terms of $(42)$ by $\omega(t)$ and integrating on $\mathcal{W}$, we then obtain

$$
\left|\left(\bar{v}_{h, x}-v(x)\right) \int_{\mathcal{W}} \omega-h \nabla v(x) \cdot \int_{\mathcal{W}} \omega(t) t d t-\frac{h^{2}}{2} \int_{\mathcal{W}} \omega(t) D^{2} v(x)(t, t) d t\right| \leq M h^{3} \int_{\mathcal{W}} \omega .
$$

Using the fact that $\operatorname{Cov}_{\mathcal{W}}(\omega)=\sigma^{2} I d$ and $\int_{\mathcal{W}} \omega(t) t d t=0$, we get

$$
\int_{\mathcal{W}} \omega(t) D^{2} v(x)(t, t) d t=\sigma^{2} \Delta v(x) \int_{\mathcal{W}} \omega
$$

and finally

$$
\bar{v}_{h, x}=v(x)+\frac{h^{2} \sigma^{2}}{2} \Delta v(x)+\underset{h \rightarrow 0}{O}\left(h^{3}\right)
$$

with $T_{h \mathcal{W}, \lambda} v(x)=\bar{v}_{h, x}$ for $h$ small enough as noticed above.

\section{$5 \quad$ Algorithm}

In this section, we propose a dual algorithm based on Chambolle's work [21], that achieves the minimization of the weighted energy

$$
E_{\lambda, \omega, v}(u)=\sum_{x \in \mathcal{W}} \omega_{x}(u(x)-v(x))^{2}+\lambda T V(u),
$$

where $\mathcal{W}$ is a finite arbitrary domain of $\mathbb{Z}^{2}, u, v$ are in $\mathbb{R}^{\mathcal{W}}$, and $\left(\omega_{x}\right) \in \mathbb{R}^{\mathcal{W}}$ are positive weights.

\subsection{Characterization of the minimizer}

Let $\mathcal{W}$ be an arbitrary subset of $\mathbb{Z}^{2}$ (not necessarily a rectangular domain). We assume as in [21] that the Total Variation is discretized according to (5), using the $\ell^{2}$-norm (9). In particular, if $y=\left(y^{1}, y^{2}\right) \in \mathbb{R}^{2}$, $|y|$ will denote its modulus, i.e. $|y|=\sqrt{\left(y^{1}\right)^{2}+\left(y^{2}\right)^{2}}$. In order to introduce the discrete divergence operator, let $\langle\cdot, \cdot\rangle$ denote the usual inner product on $\mathbb{R}^{\mathcal{W}}$, and $\langle\cdot, \cdot\rangle_{Y}$ the inner product on $\left(\mathbb{R}^{2}\right)^{\mathcal{W}}$ defined by

$$
\forall p=\left(p^{1}, p^{2}\right) \in\left(\mathbb{R}^{2}\right)^{\mathcal{W}}, \quad \forall q=\left(q^{1}, q^{2}\right) \in\left(\mathbb{R}^{2}\right)^{\mathcal{W}}, \quad\langle p, q\rangle_{Y}=\sum_{x \in \mathcal{W}}\left(p_{x}^{1} q_{x}^{1}+p_{x}^{2} q_{x}^{2}\right),
$$

the associated Euclidean norms being written $\|\cdot\|$ and $\|\cdot\|_{Y}$ respectively. Let $\delta_{i, j}^{\mathcal{W}}$ denote the real 1 if $(i, j) \in \mathcal{W}$ and 0 otherwise. If $p \in\left(\mathbb{R}^{2}\right)^{\mathcal{W}}$, the discrete divergence of $p$ is an image div $p$ defined by

$$
\forall(i, j) \in \mathcal{W}, \quad(\operatorname{div} p)(i, j)=p_{i, j}^{1} \delta_{i+1, j}^{\mathcal{W}}-p_{i-1, j}^{1} \delta_{i-1, j}^{\mathcal{W}}+p_{i, j}^{2} \delta_{i, j+1}^{\mathcal{W}}-p_{i, j-1}^{2} \delta_{i, j-1}^{\mathcal{W}}
$$

(remark that this formulation holds whatever the convention used to define $p_{x}$ when $x \notin \mathcal{W}$ ). This divergence operator div is dual to the gradient, in the sense that

$$
\forall u \in \mathbb{R}^{\mathcal{W}}, \forall p \in\left(\mathbb{R}^{2}\right)^{\mathcal{W}},\langle\operatorname{div} p, u\rangle=-\langle p, \nabla u\rangle_{Y}
$$


We also write $\|p\|_{\infty}=\max _{x \in \mathcal{W}}\left|p_{x}\right|$, and consider the (invertible) diagonal operator $D$ defined by

$$
\forall u \in \mathbb{R}^{\mathcal{W}}, \forall x \in \mathcal{W}, \quad(D u)(x)=\omega_{x} u(x),
$$

so that for any $u \in \mathbb{R}^{\mathcal{W}}$ we have $\|u\|_{\omega}=\sqrt{\sum_{x \in \mathcal{W}} \omega_{x} u(x)^{2}}=\left\|D^{1 / 2} u\right\|$. Next proposition characterizes the minimizer of (24) as the projection on a convex set.

Proposition 6 The minimizer of (43) writes

$$
T_{\omega} v=v-\pi_{\frac{\lambda}{2} K}(v)
$$

where

$$
K=\left\{D^{-1}(\operatorname{div} p), \quad p \in\left(\mathbb{R}^{2}\right)^{\mathcal{W}},\|p\|_{\infty} \leq 1\right\}
$$

is closed and convex, and $\pi_{\frac{\lambda}{2} K}$ denotes the projection operator on $\frac{\lambda}{2} K=\left\{\frac{\lambda}{2} k, k \in K\right\}$. Furthermore, if $p \in\left(\mathbb{R}^{2}\right)^{\mathcal{W}}$ is such that $\pi_{\frac{\lambda}{2} K}(v)=\frac{\lambda}{2} D^{-1}(\operatorname{div} p)$, then $p$ is characterized by

$$
\forall x \in \mathcal{W}, \quad\left|\left(\nabla\left(\frac{\lambda}{2} D^{-1}(\operatorname{div} p)-v\right)\right)_{x}\right|_{x}=\left(\nabla\left(\frac{\lambda}{2} D^{-1}(\operatorname{div} p)-v\right)\right)_{x} .
$$

For the sake of completeness, a proof derived from [21] is detailed in the Appendix . Again following [21], we can now derive an iterative scheme for the numerical minimization of (43) by considering a semi-implicit gradient descent with step $\tau>0$, given by

$$
\forall x \in \mathcal{W}, \quad p_{x}^{n+1}=p_{x}^{n}+\tau\left[\nabla\left(D^{-1} \operatorname{div} p^{n}-\frac{v}{\lambda / 2}\right)-\left|\nabla\left(D^{-1} \operatorname{div} p^{n}-\frac{v}{\lambda / 2}\right)\right| p^{n+1}\right]_{x},
$$

which leads to

$$
\forall x \in \mathcal{W}, \quad p_{x}^{n+1}=\frac{p_{x}^{n}+\tau\left(\nabla\left(D^{-1} \operatorname{div} p^{n}-\frac{v}{\lambda / 2}\right)\right)_{x}}{1+\tau\left|\nabla\left(D^{-1} \operatorname{div} p^{n}-\frac{v}{\lambda / 2}\right)_{x}\right|} .
$$

\subsection{Convergence}

\subsubsection{Main result}

When $\mathcal{W}$ is a rectangle and $\omega_{x}=1$ for all $x \in \mathcal{W}$ (i.e. $D=I d$ ), the iterative scheme (49) converges, provided that $\tau \leq 1 / 8$ [21, Theorem 3.1]. In the case of arbitrary domain $\mathcal{W}$ and arbitrary positive weights $\left(\omega_{x}\right)_{x \in \mathcal{W}}$, the scheme still converges, but the condition over the step size is modified, as stated in

Theorem 4 Let $\left(\omega_{x}\right)_{x \in \mathcal{W}}$ be positive weights on $\mathcal{W}$, and

$$
\tau_{\max }=\frac{1}{4 \max \left\{\max _{i, j}^{*}\left(\frac{1}{\omega_{i, j}}+\frac{1}{\omega_{i+1, j}}\right), \max _{i, j}^{*}\left(\frac{1}{\omega_{i, j}}+\frac{1}{\omega_{i, j+1}}\right)\right\}},
$$

the $*$ symbol in $\max _{i, j}^{*} f(i, j)$ meaning that only the indices $(i, j)$ for which $f(i, j)$ is defined are considered. Let $D$ be the linear diagonal invertible operator defined by (45), $v \in \mathbb{R}^{\mathcal{W}}$, and $\lambda>0$. For any $\tau \in\left(0, \tau_{\max }\right]$, if $\left(p^{n}\right)$ is arbitrarily initialized (with $\left.\left\|p^{0}\right\|_{\infty} \leq 1\right)$ and recursively defined by (49), then $v-\frac{\lambda}{2} D^{-1}\left(\operatorname{div} p^{n}\right)$ converges to the minimizer of (43).

Proof: We follow the proof of [21, Theorem 3.1]. For convenience, let $L$ denote the linear operator $L=D^{-1 / 2}$ div , and $L^{*}=-\nabla D^{-1 / 2}$ the dual operator. Letting $X=\mathbb{R}^{\mathcal{W}}$ and $Y=\left(\mathbb{R}^{2}\right)^{\mathcal{W}}$, note that $L \in \mathcal{L}(Y, X)$ (linear operators mapping $Y$ into $X)$ and that $L^{*} \in \mathcal{L}(X, Y)$. 
First, notice that $\left\|p^{n}\right\|_{\infty} \leq 1$ for all $n \in \mathbb{N}$, by induction on $n$ in (49). Now, denoting $\mathbf{v}=\frac{D^{1 / 2} v}{\lambda / 2}$, we prove that the sequence $\left\|L p^{n}-\mathbf{v}\right\|^{2}$ is decreasing for a certain range of values of $\tau$. Writing $\eta=$ $\left(p^{n+1}-p^{n}\right) / \tau$, we have

$$
\begin{aligned}
\| L p^{n+1} & -\mathbf{v}\left\|^{2}-\right\| L p^{n}-\mathbf{v} \|^{2} \\
& =2 \tau\left\langle L \eta, L p^{n}-\mathbf{v}\right\rangle+\tau^{2}\|L \eta\|^{2} \\
& \leq-2 \tau\left\langle\eta,-L^{*}\left(L p^{n}-\mathbf{v}\right)\right\rangle_{Y}+\tau^{2} \kappa^{2}\|\eta\|_{Y}^{2},
\end{aligned}
$$

where $\kappa$ denotes the operator norm of $L$ (an upper bound for $\kappa$ will be given at the end of the proof). Now since $\nabla\left(D^{-1} \operatorname{div} p^{n}-\frac{v}{\lambda / 2}\right)=-L^{*}\left(L p^{n}-\mathbf{v}\right)$, we get from (48) that

$$
\forall x \in \mathcal{W}, \quad \eta_{x}=-\left(L^{*}\left(L p^{n}-\mathbf{v}\right)\right)_{x}-\left|\left(L^{*}\left(L p^{n}-\mathbf{v}\right)\right)_{x}\right| p_{x}^{n+1},
$$

and consequently, for any pixel $x$, the splitting

$$
\begin{aligned}
2 \eta_{x} & \cdot\left(-L^{*}\left(L p^{n}-\mathbf{v}\right)\right)_{x} \\
& =\left|\eta_{x}\right|^{2}+\left|\left(L^{*}\left(L p^{n}-\mathbf{v}\right)\right)_{x}\right|^{2}-\left|\eta_{x}+\left(L^{*}\left(L p^{n}-\mathbf{v}\right)\right)_{x}\right|^{2} \\
& =\left|\eta_{x}\right|^{2}+\left|\left(L^{*}\left(L p^{n}-\mathbf{v}\right)\right)_{x}\right|^{2}-\left|\left(L^{*}\left(L p^{n}-\mathbf{v}\right)\right)_{x}\right|^{2}\left|p_{x}^{n+1}\right|^{2}
\end{aligned}
$$

implies that

$$
2\left\langle\eta,-L^{*}\left(L p^{n}-\mathbf{v}\right)\right\rangle_{Y} \geq\|\eta\|_{Y}^{2}
$$

because $\left\|p^{n+1}\right\|_{\infty} \leq 1$. Thus, gathering (51) and (53) yields

$$
\left\|L p^{n+1}-\mathbf{v}\right\|^{2}-\left\|L p^{n}-\mathbf{v}\right\|^{2} \leq-\tau\left(\left(1-\tau \kappa^{2}\right)\|\eta\|_{Y}^{2}\right)
$$

which is negative as soon as $\tau<1 / \kappa^{2}$. This proves that the sequence $\left\|L p^{n}-\mathbf{v}\right\|^{2}$ is decreasing, unless $\eta=0$, which anyway ensures that $p^{n+1}=p^{n}$. When $\tau=1 / \kappa^{2}$, the result remains true, because if $\left\|L p^{n+1}-\mathbf{v}\right\|=\left\|L p^{n}-\mathbf{v}\right\|$, then (53) is an equality, which requires that

$$
\forall x \in \mathcal{W}, \quad\left|\left(L^{*}\left(L p^{n}-\mathbf{v}\right)\right)_{x}\right|\left|p_{x}^{n+1}\right|=\left|\left(L^{*}\left(L p^{n}-\mathbf{v}\right)\right)_{x}\right|,
$$

so that for a given $x \in \mathcal{W}$, either $\left|p_{x}^{n+1}\right|=1$ or $\left(L^{*}\left(L p^{n}-\mathbf{v}\right)\right)_{x}=0$. In both cases, $p^{n+1}=p^{n}$ thanks to (49).

Let $m$ be the limit of $\left\|L p^{n}-\mathbf{v}\right\|$, and $\bar{p}$ be the limit of a converging subsequence $\left(p^{n_{k}}\right)$ of $\left(p^{n}\right)$. Then by $(49),\left(p^{n_{k}+1}\right)$ converges to a certain $\bar{p}^{\prime}$ such that

$$
\forall x \in \mathcal{W}, \quad \bar{p}_{x}^{\prime}=\frac{\bar{p}_{x}+\tau\left(L^{*}(L \bar{p}-\mathbf{v})\right)_{x}}{1+\tau\left|\left(L^{*}(L \bar{p}-\mathbf{v})\right)_{x}\right|},
$$

and repeating the former computations leads, thanks to the fact that $m=\|L \bar{p}-\mathbf{v}\|=\left\|L \bar{p}^{\prime}-\mathbf{v}\right\|$, to $\bar{\eta}=\left(\bar{p}^{\prime}-\bar{p}\right) / \tau=0$, that is, $\bar{p}=\bar{p}^{\prime}$. Thus, taking the limit $\left(n=n_{k}, k \rightarrow+\infty\right)$ in (52), we get

$$
\forall x \in \mathcal{W}, \quad-\left(L^{*}(L \bar{p}-\mathbf{v})\right)_{x}=\left|\left(L^{*}(L \bar{p}-\mathbf{v})\right)_{x}\right| \bar{p}_{x}
$$

which precisely characterizes the minimizer of (43), as shown in Proposition 6 . Hence, $\frac{\lambda}{2} D^{-1} \operatorname{div} \bar{p}$ is the projection $\pi_{\frac{\lambda}{2} K}(v)$. Since this projection is unique, we deduce that the whole sequence $\left(\frac{\lambda}{2} D^{-1} \operatorname{div} p^{n}\right)_{n}$ tends to the desired projection, and consequently, thanks to Proposition 6, that $\left(v-\frac{\lambda}{2} D^{-1} \operatorname{div} p^{n}\right)_{n}$ converges towards the minimizer of (43).

Now we compute an upper bound for the norm $\kappa$ of the operator $L$. We have, for any $\eta$,

$$
\begin{aligned}
\|L \eta\|^{2}=\left\|D^{-1 / 2} \operatorname{div} \eta\right\|^{2} & =\sum_{(i, j) \in \mathcal{W}} \frac{1}{\omega_{i, j}}\left(\eta_{i, j}^{1} \delta_{i+1, j}^{\mathcal{W}}-\eta_{i-1, j}^{1} \delta_{i-1, j}^{\mathcal{W}}+\eta_{i, j}^{2} \delta_{i, j+1}^{\mathcal{W}}-\eta_{i, j-1}^{2} \delta_{i, j-1}^{\mathcal{W}}\right)^{2} \\
& \leq \sum_{(i, j) \in \mathcal{W}} \frac{4}{\omega_{i, j}}\left[\left(\eta_{i, j}^{1} \delta_{i+1, j}^{\mathcal{W}}\right)^{2}+\left(\eta_{i-1, j}^{1} \delta_{i-1, j}^{\mathcal{W}}\right)^{2}+\left(\eta_{i, j}^{2} \delta_{i, j+1}^{\mathcal{W}}\right)^{2}+\left(\eta_{i, j-1}^{2} \delta_{i, j-1}^{\mathcal{W}}\right)^{2}\right] \\
& \leq 4 \sum_{i, j} *\left(\frac{1}{\omega_{i, j}}+\frac{1}{\omega_{i+1, j}}\right)\left(\eta_{i, j}^{1}\right)^{2}+4 \sum_{i, j}^{*}\left(\frac{1}{\omega_{i, j}}+\frac{1}{\omega_{i, j+1}}\right)\left(\eta_{i, j}^{2}\right)^{2},
\end{aligned}
$$


where the notation $\sum_{i, j}^{*} f(i, j)$ means, as in Theorem 4, that only the indices $(i, j)$ for which $f(i, j)$ is defined are considered. This provides the upper bound

$$
\kappa^{2} \leq 4 \max \left\{\max _{i, j} *\left(\frac{1}{\omega_{i, j}}+\frac{1}{\omega_{i+1, j}}\right), \max _{i, j} *\left(\frac{1}{\omega_{i, j}}+\frac{1}{\omega_{i, j+1}}\right)\right\},
$$

so that, taking $\tau \leq \tau_{\max }$ (with $\tau_{\max }$ as in (50)) yields the announced result.

Remark 1 (maximum upper bound). In Section 4.3.2, we decided to normalize the weights by taking $\omega_{0}=1$. If in addition we suppose that $\omega_{0}$ is the maximum weight (which is quite natural), then we have $\max _{x \in \mathcal{W}} \omega_{x}=1$ and consequently $\tau_{\max } \leq 1 / 8$. Note that the equality $\tau_{\max }=1 / 8$ (which is the bound given in [21]) is only reached for uniform weights $(\omega \equiv 1)$.

Remark 2 (practical convergence). In numerical experiments where $\omega \equiv 1$, the effective maximal step size allowing convergence is $1 / 4$ [21], that is, twice the limit $\tau_{\max }$ predicted by the theory (Aujol [9] gives an explanation for this, derived from Bermúdez-Moreno's algorithm). For Gaussian weights (28), we observed that the effective maximum step size $\tau_{\max }^{\text {eff }}$ satisfied

$$
\tau_{\max }^{\text {eff }} \in\left[2 \tau_{\max }, 4 \tau_{\max }\right] .
$$

The value $\tau_{\max }^{\text {eff }} \simeq 4 \tau_{\max }$ is found when $\mathcal{W}$ is much larger than the kernel's bandwidth, so that the Gaussian kernel is hardly truncated by the boundary of $\mathcal{W}$. Conversely, when $\mathcal{W}$ is much smaller than the kernel bandwidth, the weights are virtually uniform on $\mathcal{W}$, and $\tau_{\max }^{\text {eff }} \simeq 2 \tau_{\max }$. In the latter case, however, aliasing-like effect is to be expected (as shown in Section 3.2), so that a trade-off has to be found between the complete removal of the artifact and the speed of the algorithm.

\subsubsection{Pointwise convergence control}

Adapting Chambolle's criterion [22] to our case, we are able to explicitly control the weighted $L^{2}$-distance between the optimum denoised patch and the patch coming from the iterations of the algorithm. Indeed, let $u^{n}=v-\frac{\lambda}{2} D^{-1} \operatorname{div} p^{n}$ be the image obtained after the $n$-th iteration. Let also $\bar{p}$ denote the limit of $p^{n}$, and $\bar{u}=v-\frac{\lambda}{2} D^{-1} \operatorname{div} \bar{p}$. Then,

$\left\|u^{n}-\bar{u}\right\|_{\omega}^{2}=\left\langle\frac{\lambda}{2} D^{-1}\left(\operatorname{div} \bar{p}-\operatorname{div} p^{n}\right), D\left(u^{n}-\bar{u}\right)\right\rangle=\frac{\lambda}{2}\left\langle\operatorname{div} \bar{p}-\operatorname{div} p^{n}, u^{n}-\bar{u}\right\rangle=\frac{\lambda}{2}\left\langle p^{n}-\bar{p}, \nabla u^{n}-\nabla \bar{u}\right\rangle_{Y}$.

Now $\left|\langle p, \nabla u\rangle_{Y}\right| \leq T V(u)$ for every $u \in \mathbb{R}^{\mathcal{W}}$ and for every $p \in\left(\mathbb{R}^{\mathcal{W}}\right)^{2}$ satisfying $\|p\|_{\infty} \leq 1$. But as $\langle\bar{p}, \nabla \bar{u}\rangle_{Y}=-T V(\bar{u})$ (consider Equation 47 with $\frac{\lambda}{2} D^{-1} \operatorname{div} \bar{p}=v-\bar{u}$ ), we get

$$
\left\|u^{n}-\bar{u}\right\|_{\omega}^{2} \leq \frac{\lambda}{2}\left(T V\left(u^{n}\right)+\left\langle p^{n}, \nabla u^{n}\right\rangle_{Y}\right),
$$

which is a computable local convergence bound (the right-hand term going to 0 as $n \rightarrow+\infty$ ), that entails the pointwise convergence criterion

$$
\forall x \in \mathcal{W}, \quad\left|u^{n}-\bar{u}\right|^{2}(x) \leq \frac{\lambda}{2}\left(T V\left(u^{n}\right)+\left\langle p^{n}, \nabla u^{n}\right\rangle_{Y}\right)
$$

since $\omega_{0}=1$.

\subsubsection{Interest of considering an arbitrary neighborhood shape}

The first main interest of an arbitrary neighborhood shape is to speed up the algorithm. Indeed, the pixels $x$ corresponding to negligible values of the weight $\omega_{x}$ can be removed from the neighborhood shape 
without changing much the associated local TV-filter, and this results in an arbitrary neighborhood shape with an increased value of $\tau_{\max }$ (which speeds up the algorithm).

A second important interest is to be able to deal with adaptive neighborhoods. A typical shape to be used for $\mathcal{W}$ is a discrete ball with a fixed radius, so that the algorithm becomes more isotropic. But the neighborhoods can also be designed adaptively with respect to the image: not only the radius can be adapted to the local scale of the image, but also the shape could be distorted along the geometry of the image as in [42]. We shall focus on different applications in the remaining part of this paper, but these ideas could be interesting directions to explore.

\section{Experiments with local TV-denoising}

In this section, the local TV-filter considered in Definition 2 is directly used as a denoising filter. All denoising experiments that follow are computed with the iterative algorithm described in Section 5. Basically, this algorithm is not very fast because it requires the minimization of a local energy for each pixel. Hopefully, we can take advantage of the photometric similarity between successive image patches (according to the lexicographic order on pixels) to speed up the minimization process. Indeed, as TVdenoising is non-expansive (20), the spatial coherence of the image implies that the solution associated to a given pixel is necessarily close to the solution associated to its four adjacent pixels. Thus, by initializing the algorithm for each pixel with the solution found for the previous pixel, we start the iterative process with a much better initial guess than a classical initialization (e.g. a constant image), and the convergence is attained much more quickly.

Concerning the processing of image borders, we adopted a classical solution that consists in extending the image domain with a symmetry convention. This permits to maintain, for each pixel of the original image domain, the same neighborhood shape. Another solution would have been to intersect the theoretical neighborhood of each pixel with the image domain, but such a process is likely to artificially favor a linear denoising near the image borders in reason of the decrease of the neighborhood area (see Section 4.4.2).

\subsection{Locality of global TV}

Now that a local version of TV-denoising has been proposed, we are able to confirm by numerical experiments that global TV-denoising is mainly ruled by local interactions (see Section 2). In Figure 4, several images denoised by local TV are compared using the $L^{2}$ norm to the ones denoised by global $\mathrm{TV}$, for all sizes of square neighborhoods $\mathcal{W}$ smaller than $19 \times 19$ (and constant weight functions in all neighborhoods). As predicted in Section 2, the locally denoised images quickly converge to the globally denoised ones, when the neighborhood size increases: in practice a $11 \times 11$ patch is sufficient to capture most of the interactions, which means that long-range interactions caused by global TV-denoising are globally negligible in these ordinary images. It is also interesting to notice that the curves more or less coincide. This relative stability among images is likely to make the choice of the window size easier.

\subsection{Local versus global denoising, bias-variance trade-off}

Consider the local TV-filter associated to a Gaussian weight function with standard deviation $a$ (written $\omega^{a}$ ), as in Equation (28). When the locality parameter $a$ is small, local TV-denoising is approximately equivalent to linear filtering; inversely, it is equivalent to global TV-denoising when $a$ is large (see paragraph at the end of Section 4.2). For intermediate values of $a$, local TV-denoising may achieve an interesting compromise between these two extreme behaviors, as illustrated in Figure 5 on a synthetic triangle signal. Indeed, the intermediate local TV-denoising model is able to combine a good restoration of intensity gaps (edges) with a regular smoothing of the regular parts while limiting the cropping of the 

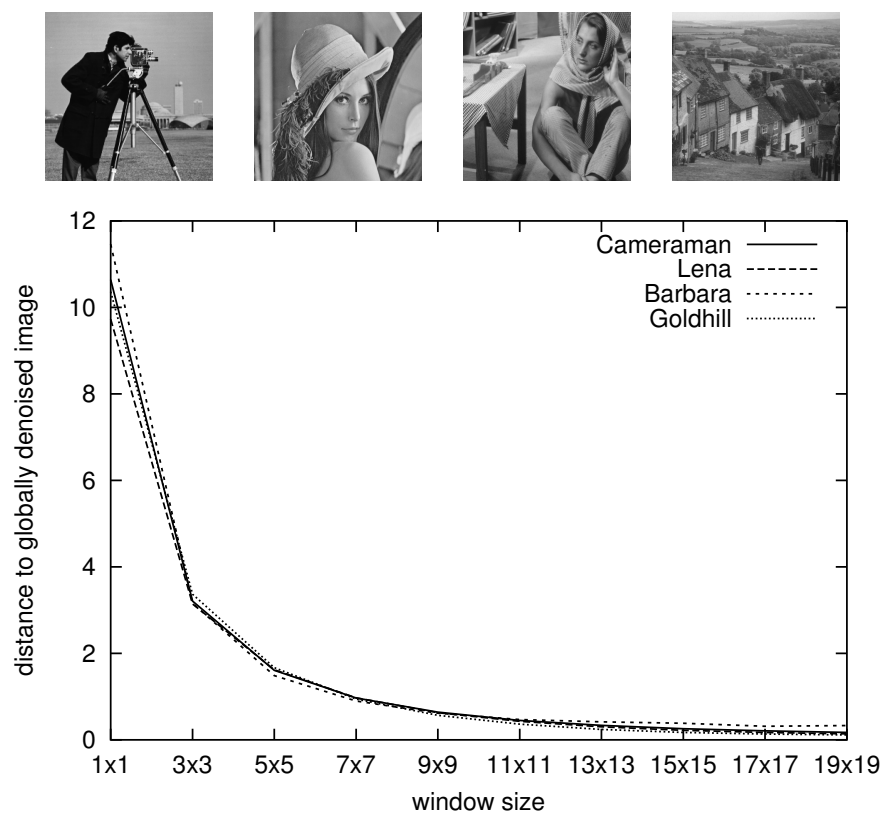

Figure 4: From local to global denoising. For each of the 4 classical images Cameraman, Lena, Barbara and Goldhill, degraded by an additive white Gaussian noise $(\sigma=10)$, the graph shows the quick decrease of the $L^{2}$-distance between the globally denoised image and the locally denoised one using increasing window sizes (hard window, and constant $\lambda=20$ ). Despite the fact that the image contents are very different (miscellaneous textures and different scales), the convergence curves above seem quite independent of the image.

extrema. It also avoids the staircasing artifact occurring in global TV-denoising, as we shall see more precisely in Section 6.3.

From a statistical viewpoint, this compromise can be seen as a bias-variance trade-off with respect to the locality parameter $a \in(0, \infty)$, the regularization parameter $\lambda$ being fixed. Indeed, if $\varepsilon$ is a (white) noise process, the mean square error made by local TV-denoising can be classically decomposed into

$$
\mathbb{E}_{\varepsilon}\left\|T_{\omega^{a}, \lambda}(u+\varepsilon)-u\right\|^{2}=\underbrace{\left\|\mathbb{E}_{\varepsilon}\left[T_{\omega^{a}, \lambda}(u+\varepsilon)\right]-u\right\|^{2}}_{\text {squared bias }}+\underbrace{\mathbb{E}_{\varepsilon}\left\|T_{\omega^{a}, \lambda}(u+\varepsilon)-\mathbb{E}_{\varepsilon}\left[T_{\omega^{a}, \lambda}(u+\varepsilon)\right]\right\|^{2}}_{\text {variance }} .
$$

When $a$ is close to 0 , local TV-denoising is close to the identity operator, whose bias is zero but whose variance is equal to the noise variance. Conversely, when $a$ is large, local TV-denoising is close to global TV-denoising, that has a much smaller variance but a large bias (in particular because it kills extrema, as shown in Figure 5, up-right). The bias and variance terms do not seem to follow a simple law with respect to $a$, but in general the best compromise is found for a finite (non-zero) value of $a$, as illustrated in Figure 5.

\subsection{Reduction of the staircasing effect}

In this paragraph we show how local TV-denoising is able to circumvent a strong drawback of global TV-denoising. Indeed, an image denoised by global TV-denoising tends to contain blocks with constant gray level, separated by intensity gaps (edges), even in what should be smooth areas. This so-called "staircasing effect" also occurs in other methods such as denoising by neighborhood filters [16], and in image or video compression methods.

The staircasing effect in global TV-denoising was first reported by Dobson and Santosa [35] and used to denoise piecewise constant images. The first mathematical proofs for its existence were successively 


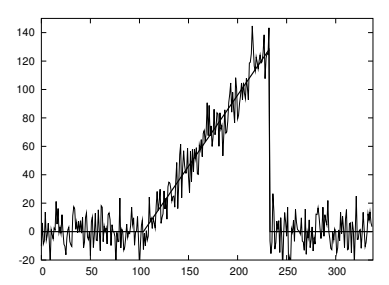

noisy triangle

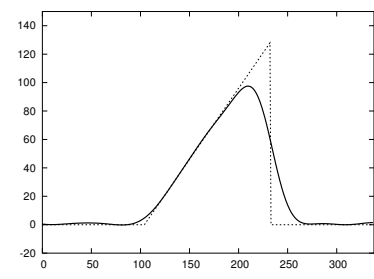

Gaussian filtering $(a=15)$

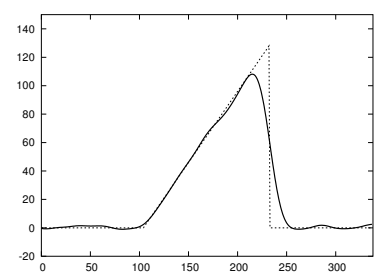

Gaussian filtering $(a=10)$

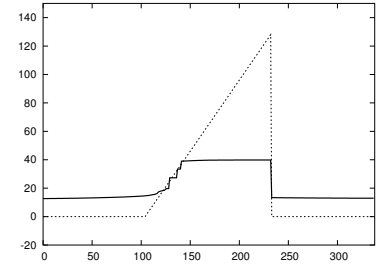

global TV $(\lambda=4000)$

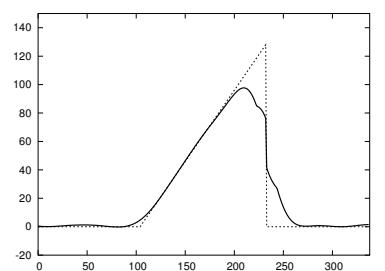

local TV $(a=15, \lambda=4000)$

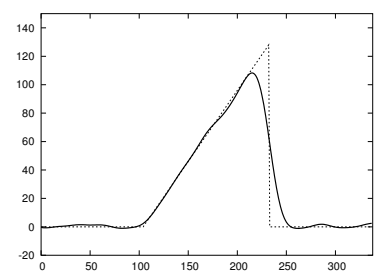

local TV $(a=10, \lambda=4000)$

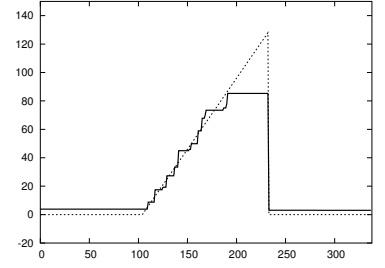

global TV $(\lambda=1000)$

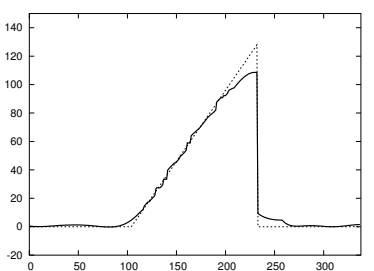

local TV $(a=15, \lambda=1000)$

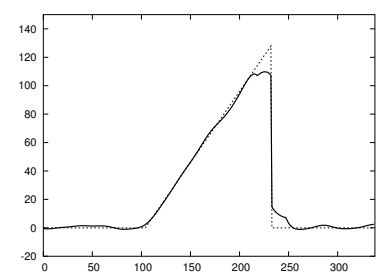

local TV $(a=10, \lambda=1000)$

Figure 5: A triangle signal corrupted by an additive white Gaussian noise with $\sigma=10$ (top, left) is denoised by three different algorithms, that all correspond to particular cases of local TV-denoising. The global TV method (top row), which corresponds to $a=+\infty$, preserves the original "discontinuity" (that is, the presence of an intensity gap) but largely destroys the maximum intensity zone and creates staircasing artifacts. The Gaussian filtering (first column), which corresponds to $\lambda=+\infty$, avoids staircasing and extrema killing but introduces a lot of blur that completely loses the original intensity gap. An interesting compromise between these two methods can be obtained by using local TV-denoising with finite values of $a$ and $\lambda$, as can be seen on the four bottom-right images $(2 \times 2$ square $)$, and in particular the two rightmost images $(\lambda=1000)$.

given by Nikolova [48, 49] and Ring [53] in different contexts. Theoretical research about the staircasing artifact is active, as show [47] and [19] for instance. Several methods were proposed to address this issue: Chambolle and Lions [20], Blomgren et al. [13] and Levine et al. [44] propose to consider modifications of $T V$ for small gradient intensities. Chan et al. [24, 27] propose to use higher-order terms to capture the smooth regions. In [45], the authors propose to use the ROF energy in a mean-square error sense, which annihilates the staircasing effect.

The local TV-filter we proposed here is naturally free from staircasing, because thanks to Proposition 5 (Section 4.4.1), when $v$ is locally flat enough, local TV-denoising is equivalent to a blur by a low-pass filter, which naturally avoids the creation of spurious edges in smooth regions. In Figure 6, we can observe in the denoised images that contrasted edges are well preserved as in global TV-denoising, while smooth regions are much more faithfully reconstructed.

Despite the fact that local TV-denoising visually improves global TV-denoising in a significant way, it still suffers from the inability of Total Variation to cope with textures, that are not distinguished from noise and hence systematically "washed out" by TV-based methods. Even if this drawback can be ignored for some kinds of images (in particular scientific images aiming at measuring geometrical features), it puts TV-based denoising methods a step behind state-of-the-art denoising methods. However, as we shall see now, having transformed TV-denoising into a local filter opens interesting perspectives for efficient image denoising, in particular in combination with the recent NL-means algorithm [15]. 

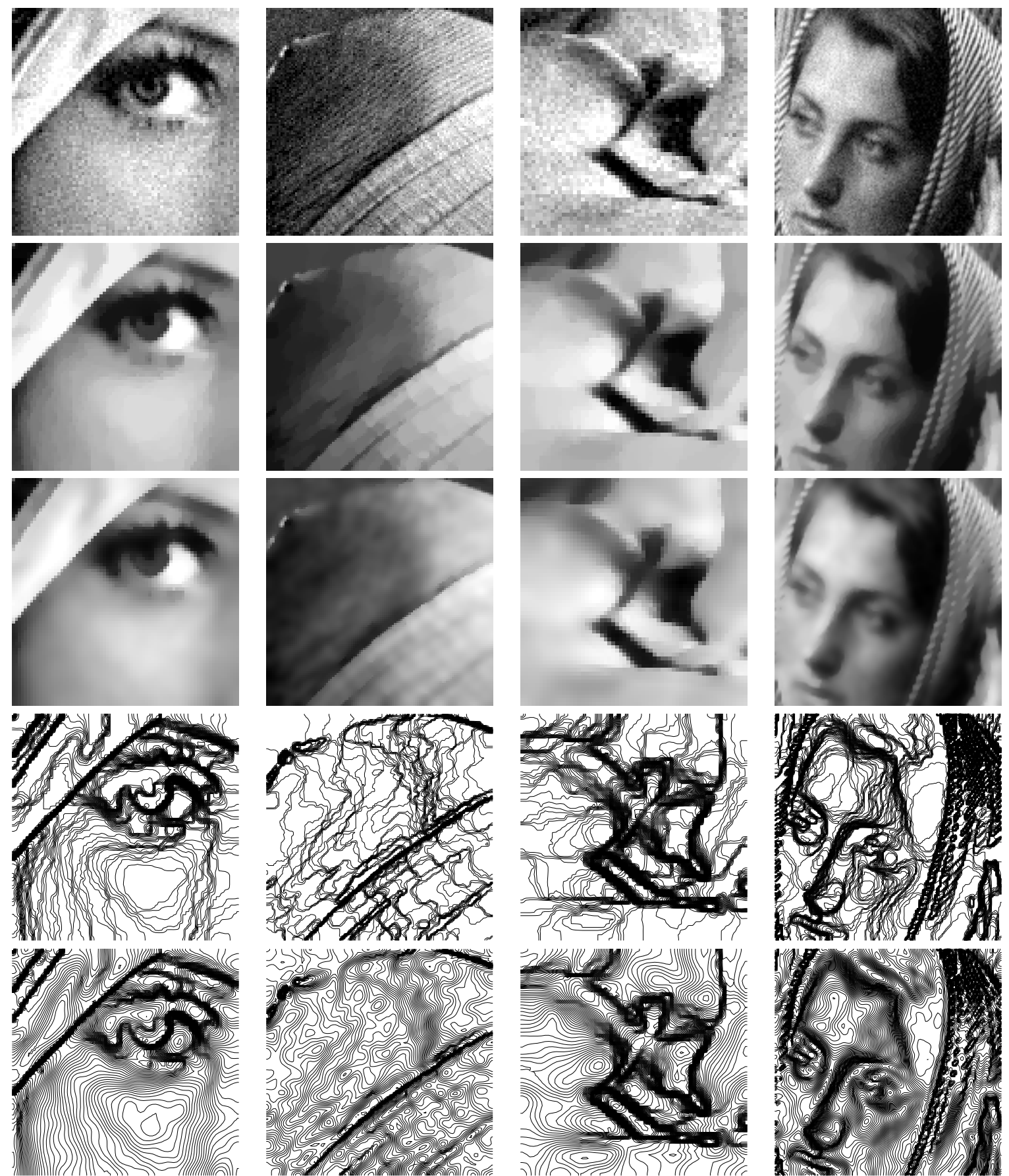

Figure 6: Removal of the staircasing artifact. On each column, the noisy image $(\sigma=10)$ of the first row is denoised using global TV (second row) and local TV (third row) with $a=2$ (smooth $13 \times 13$ window), both for $\lambda=40$. As we can see, the staircasing effects (artificial edges) that appear with global TV-denoising completely disappear with local TV. This is confirmed on rows 4 and 5 (that display the level lines of the images of rows 2 and 3 respectively): instead of being artificially grouped together due to the staircasing effect (row 4), the level lines in smooth areas are much more regularly spaced in the case of local TV-denoising (row 5). 


\section{Application to a hybrid TV-NLmeans denoising filter}

In this part, we show how the local TV-filter we studied and the Non-Local means (NL-means) denoising algorithm [15] can be combined together to build a new denoising filter that significantly improves the two methods taken separately. The NL-means algorithm, recently introduced in the continuity of the bilateral filter [58,60] and patch-based models for inpainting or texture synthesis [29, 37], has shown how the redundancy of image patches could be used to achieve very interesting denoising performances, especially in textured areas. For that reason, its combination with TV-denoising, whose principal drawback is its inability to deal with textures, seems natural. Note that our goal here is simply to show that the combination of two very different principles (patch-based denoising and TV-regularization) can lead to interesting new denoising algorithms; finding the best possible combination would require a dedicated study that cannot be done here.

\section{$7.1 \quad$ NL-means}

Whereas classical local filters build an estimate of the true (that is, noise-free) gray level at a given pixel $x$ by averaging gray levels of pixels $y$ that are located near $x$ (that is, for which $|y-x|$ is small), the NL-means algorithms uses pixels $y$ that are similar to $x$, in the sense that the image patches $u\left(\mathcal{N}_{x}\right)$ and $u\left(\mathcal{N}_{y}\right)$ are similar (here $\mathcal{N}$ is a fixed patch shape, typically a $7 \times 7$ square). More precisely, an image $v \in \mathbb{R}^{\Omega}$ is denoised by

$$
\operatorname{NLmeans}(v)(x)=\frac{\sum_{y \in \Omega} \omega_{x, y} v(y)}{\sum_{y \in \Omega} \omega_{x, y}},
$$

where each weight

$$
\omega_{x, y}=\exp \left(-\frac{d\left(v\left(\mathcal{N}_{x}\right), v\left(\mathcal{N}_{y}\right)\right)^{2}}{2 h^{2}}\right)
$$

is a similarity measure between the patches $v\left(\mathcal{N}_{x}\right)$ and $v\left(\mathcal{N}_{y}\right)$, based on the Gaussian-weighted Euclidean distance

$$
d\left(v\left(\mathcal{N}_{x}\right), v\left(\mathcal{N}_{y}\right)\right)=\left(\frac{\sum_{k \in \mathcal{N}} \alpha_{k}(v(x+k)-v(y+k))^{2}}{\sum_{k \in \mathcal{N}} \alpha_{k}}\right)^{\frac{1}{2}}
$$

with $\alpha_{k}=e^{-|k|^{2} /\left(2 a^{2}\right)}$ and $a \in(0,+\infty]$ (the case $a=\infty$ corresponds to the unweighted case). Using a weighted norm in the patch comparisons is a small improvement that permits to give more importance to values in the center of the patch. A reasonable choice for $a$ is $a=\frac{s-1}{4}$ for a $s \times s$ patch, so that the Gaussian weight varies in $\left[e^{-4}, e^{-2}\right] \simeq[0.018,0.05]$ on the patch boundary. For computational reasons, the exploration domain of $y$ occurring in the sums of (57) is generally restricted to a (not so large) neighborhood $\mathcal{W}_{x}$ of $x$. This makes the NL-means algorithms less "non-local", but brings a significant improvement, in terms of computational time and denoising performances.

The efficiency of the NL-means method comes from the fact that most image pixels of a natural image have similar image pixels (in the sense defined above). Let us be a little more precise and say that a patch $v\left(\mathcal{N}_{y}\right)$ is a replica of $v\left(\mathcal{N}_{x}\right)$ if they both come from the same original (noise-free) patch to which independent noises have been added. If the patch $v\left(\mathcal{N}_{y}\right)$ is a replica of $v\left(\mathcal{N}_{x}\right)$ and $\mathcal{N}_{x} \cap \mathcal{N}_{y}=\emptyset$, then we have

$$
d\left(v\left(\mathcal{N}_{x}\right), v\left(\mathcal{N}_{y}\right)\right)^{2}=2 \sigma^{2} \frac{\sum_{k \in \mathcal{N}} \alpha_{k} Z_{k}^{2}}{\sum_{k \in \mathcal{N}} \alpha_{k}},
$$

where $\left(Z_{k}\right)$ are i.i.d. random variables with distribution $\mathcal{N}(0,1)$. In particular we have

$$
\mathbb{E}\left[d\left(v\left(\mathcal{N}_{x}\right), v\left(\mathcal{N}_{y}\right)\right)^{2}\right]=2 \sigma^{2},
$$

and the classical choice $h=\sigma$ ensures that most replicas have a non-negligible weight $\omega_{x, y}$. 
The main weakness of NL-means denoising is probably the way it handles exceptional patches, that is, image patches $v\left(\mathcal{N}_{x}\right)$ for which $d\left(v\left(\mathcal{N}_{x}\right), v\left(\mathcal{N}_{y}\right)\right)^{2} \gg 2 \sigma^{2}$ for almost all pixels $y$. In reason of the definition of the weights $\omega_{x, y}$ (Equation 58) and the fact that $\omega_{x, x}=1$, the NL-means algorithm will either leave a lot of noise (for small values of $h$ ) or produce an exaggerated blur (for larger values of $h$ ). This last effect comes from the fact that the averaging is done with pixel values coming from quite different patches, leading to a "patch jittering" blur effect. In practice, this phenomenon is commonly observed on images processed with the NL-means algorithm: a significant amount of noise is left around edges (and other local image structures like corners, T-junctions,etc.), and several image parts suffer from a noticeable blur effect. An appropriate choice of the parameter $h$ permits to reduce any of these two effects, but not both simultaneously. Another consequence of this "patch jittering" blur effect is the fact that when the patch size gets larger, the NL-means filter tends to produce more blurry images (see Figure 7, right column), although intuitively, the decreasing number of similar patches should lead to a lower level of denoising, producing a noise effect.

\subsection{A new filter: TV-means}

In order to take care of exceptional patches, we propose to change the NL-means strategy in two ways:

(A) select only patches that could be replica of the current patch;

(B) if the number of selected patches is too small, apply TV-regularization.

The step (A) was proposed before in [14, 31, 46, 50], and, in a different way, in [43]. Notice that the strategy $(\mathrm{A})+(\mathrm{B})$ we propose simply consists in smoothing exceptional patches with a well-established method (TV-denoising) instead of using the indirect "patch jittering" smoothing effect. This combination of local (patch) TV-denoising and NL-means yields the following algorithm called TV-means.

For a given patch shape $\mathcal{N}$, a given search window $\mathcal{W}$ (as in NL-means), and a threshold $\tau$ (that will be specified afterwards), let us define, for every $\lambda \geq 0$, the set

$$
\Omega(x, \lambda)=\left\{y \in \mathcal{W}_{x}, d\left(T_{\lambda}\left(v\left(\mathcal{N}_{x}\right)\right), T_{\lambda}\left(v\left(\mathcal{N}_{y}\right)\right)\right)^{2}<\tau\right\},
$$

estimating the location of possible replicas of $v\left(\mathcal{N}_{x}\right)$ after TV-filtering with parameter $\lambda$. Let now $\left(n_{\lambda}\right)_{\lambda \geq 0}$ denote a nonincreasing sequence (this sequence controls the level of denoising and will be discussed afterwards). To every $x \in \Omega$, we associate

$$
\hat{\lambda}(x)=\min \left\{\lambda \geq 0,|\Omega(x, \lambda)| \geq n_{\lambda}\right\}
$$

which represents the minimal TV-filtering parameter for which $T_{\lambda}\left(v\left(\mathcal{N}_{x}\right)\right)$ has enough (that is, at least $\left.n_{\lambda}\right)$ replicas. We finally denoise the pixel $x$ by averaging the local TV-denoising estimates, that is

$$
\operatorname{TVmeans}(v)(x)=\frac{1}{|\Omega(x, \hat{\lambda}(x))|} \sum_{y \in \Omega(x, \hat{\lambda}(x))} T_{\hat{\lambda}(x)}^{\mathcal{N}} v(y) .
$$

Threshold on the patch distance. The threshold $\tau$ is chosen such that a patch $v\left(\mathcal{N}_{x}\right)$ and one of its replicas $v\left(\mathcal{N}_{y}\right)$ have a probability of 0.99 to be considered as similar. Remembering that $v\left(\mathcal{N}_{y}\right)-v\left(\mathcal{N}_{x}\right)$ is a Gaussian random variable and assuming that the patch size is large enough, we can use a result from Fisher [39] giving a central limit theorem on weighted i.i.d. random variables and write, as $\left(Z_{x}^{2}-1\right) / \sqrt{2}$ are centered and normalized i.i.d. random variables,

$$
\frac{\sum_{x \in \mathcal{N}} \alpha_{x} \frac{Z_{x}^{2}-1}{\sqrt{2}}}{\sqrt{\sum_{x \in \mathcal{N}} \alpha_{x}^{2}}} \sim \mathcal{N}(0,1)
$$


Hence, combining (60) and (61) yields

$$
d\left(v\left(\mathcal{N}_{x}\right), v\left(\mathcal{N}_{y}\right)\right)^{2} \sim 2 \sigma^{2}\left(1+\frac{\sqrt{2 s_{2}}}{s_{1}} \mathcal{N}(0,1)\right) \quad \text { where } s_{p}=\sum_{k \in \mathcal{N}} \alpha_{k}^{p} \quad(p=1,2) .
$$

A look on the cumulative function of the normal distribution tables gives

$$
\mathbb{P}\left(d\left(v\left(\mathcal{N}_{x}, v\left(\mathcal{N}_{y}\right)\right)^{2}<\tau\right)=0.99 \Longleftrightarrow \tau \approx 2 \sigma^{2}\left(1+2.33 \frac{\sqrt{2 s_{2}}}{s_{1}}\right)\right.
$$

which is the value of $\tau$ that we choose to define $\Omega(x, \lambda)$.

Required number of patches for each scale. Here we explain how the sequence $\left(n_{\lambda}\right)_{\lambda \geq 0}$ (the minimum number of replicas required for a TV-filtering scale $\lambda$ ) can be defined in a generic way. The first term $n_{0}$ represents the minimal number of replicas to be found in order to avoid TV-filtering. It is a parameter of the TV-means algorithm, that sets the balance between the TV and NL-means combination. In practice a small value is convenient, and we chose $n_{0}=10$ in all experiments (and $n_{0}=6$ in the aggregated case explained in the next section).

Then, we set the other terms $n_{\lambda}(\lambda>0)$ in order to approximately keep the same level of denoising, whatever $\lambda$, that is,

$$
\forall \lambda \geq 0, \quad \frac{1}{n_{0}} \mathbb{E}\left[(v(x)-u(x))^{2}\right]=\frac{1}{n_{\lambda}} \mathbb{E}\left[\left(T_{\lambda}^{\mathcal{N}} v(x)-u(x)\right)^{2}\right]
$$

where $u$ denotes the original noise-free image (recall that averaging $n$ replicas divides the noise variance by $n$ ). Now, simulations show that the approximation

$$
\mathbb{E}\left[\left(T_{\lambda}^{\mathcal{N}} v(x)-u(x)\right)^{2}\right] \approx \sigma^{2}(1-r \lambda)
$$

where $r \in[0.05,0.1]$, usually holds for relatively small values of $\lambda$, and is quite stable with respect to $\sigma$. This justifies the choice of

$$
n_{\lambda}=n_{0}(1-r \lambda),
$$

with $r=0.1$, since we do not want to oversmooth exceptional patches. This formula was taken in all experiments of Section 7.4.

\subsection{Aggregated TV-means}

A very simple improvement that can be brought to the TV-means algorithm consists in taking advantage of the fact that for each pixel $x$, we can use the algorithm described in Section 5 to estimate not only a value for the pixel $x$, but a complete denoised patch

$$
\hat{u}_{x}: k \in \mathcal{N} \mapsto \frac{1}{|\Omega(x, \hat{\lambda}(x))|} \sum_{y \in \Omega(x, \hat{\lambda}(x))} T_{\hat{\lambda}(x)}\left(v\left(\mathcal{N}_{y}\right)\right)(y+k) .
$$

All these estimate of $u_{0}(x)$ (the non-observed noise-free image at point $x$ ), obtained for all patches containing the pixel $x$, can then be aggregated to define the denoised image

$$
x \mapsto \frac{\sum_{k \in \mathcal{N}} \alpha_{k} \hat{u}_{x+k}(-k)}{\sum_{k \in \mathcal{N}} \alpha_{k}},
$$

that significantly improves the denoising process. We call this variant "aggregated TV-means".

Since the effect of the weights $\alpha_{k}$ was very small (and not systematically improving the results), we chose constant weights $\left(\alpha_{k}=1\right.$ for all $k$ ) in all experiments, both for the aggregated and non-aggregated variants of TV-means. In addition to a simpler formulation (and the removal of the $a$ parameter), it has the advantage of speeding up a little the algorithms (with no weight, the same kind of denoising is obtained with a smaller patch size). 


\subsection{Experiments}

The TV-means and aggregated TV-means methods are straightforward to implement. However, it is worth mentioning that a considerable acceleration can be obtained by avoiding redundant computations of local TV-filtering. For a given patch $\mathcal{N}_{x}$ and a given scale $\lambda$, the computation of $T_{\lambda}\left(v\left(\mathcal{N}_{x}\right)\right)$ is done at most once, and the smoothed patch is stored until it is out of range of the remaining potential patches (the image is processed by increasing line numbers, so that the pixel $x$ cannot be part of a search window after a certain line index is reached). Using this implementation (in C language), the application of the aggregated TV-means filter takes 12 seconds on a recent PC desktop for a $256 \times 256$ image (for the patch and search window sizes specified below).

We tested the effect of TV-means denoising (in both original and aggregated variants) on the five classical images used in [43]: Barbara, Lena, Boats, House and Peppers. These five images were corrupted with a white Gaussian noise (standard deviation $\sigma=20$ ), then processed with the algorithms considered above. Concerning the choice of the parameters, we tried to find, for each algorithm, a set of values that yielded good results (in terms of PSNR, see below) for all five images:

- for global TV (ROF) denoising, we used the value $\lambda=28$;

- for NL-means, we used $7 \times 7$ patches on a $11 \times 11$ search window, a patch norm coefficient $a=$ $\frac{s-1}{4}=1.5$, and a weight decay $h=18$;

- for TV-means (without aggregation), we used $11 \times 11$ patches on a $15 \times 15$ search window, with $n=10$

- for aggregated TV-means, we used $n=6$ (since the aggregation process is a smoothing process, it is logical to be less demanding on $n$ ) and the other parameters as in the non-aggregated case.

These 4 algorithms are compared visually on Figure 7 and 8 , where a part of the images Barbara and Lena is shown. The interest of the combination of global TV-denoising and NL-means denoising clearly appears: the TV-means method, in both variants, manages to capture the best features of its two basic components: the patch redundancy used in NL-means, and the nice denoising of edges of global TV-denoising.

We also systematically evaluated the algorithms in terms of their ability to restore the original image. In each case, we computed the Peak Signal to Noise Ratio (PSNR) between the groundtruth image $u_{0}$ and the denoised image $v$, defined by

$$
P S N R=10 \log _{10}\left(\frac{255^{2}}{\sum_{x}\left(v(x)-u_{0}(x)\right)^{2}}\right) .
$$

The values are reported on Table 1. As we can observe, in terms of denoising efficiency (high PSNR), the aggregated TV-means method performs significantly better than global TV (ROF) and NLmeans, and yield performances similar to Kervrann et al. [43] results (better for Barbara, House and Peppers, and a little bit worse for Lena and Boats). Knowing that contrary to Kervrann et al. algorithm, the method we propose does not try to optimize locally the patch size, this is a promising result, since there probably are several interesting ways to combine in a more sophisticated manner the local TV and NL-means filtering. On Table 1, we also mentioned another recent method, BM3D [31], that shows state-of-the-art performances. It could be interesting to build an algorithm using the TV-means basic idea in combination with several ingredients of BM3D (two-pass denoising, collaborative stack patch filtering, etc.). Even the idea of any-shape patches, used in the recent Shape-Adaptive PCA BM3D [32], could be combined with local TV-denoising thanks to the general framework we used in Section 3 to define local TV-filtering on an arbitrary neighborhood shape. 


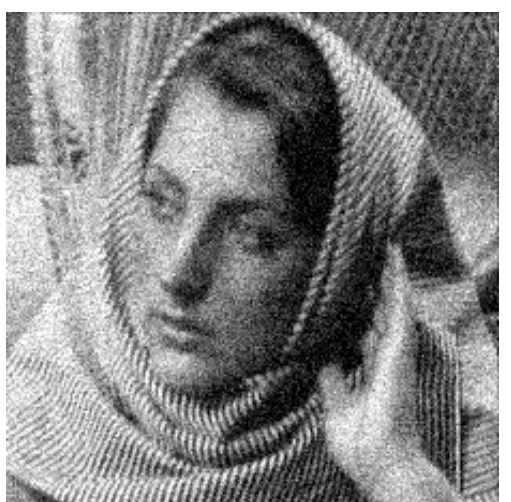

noisy

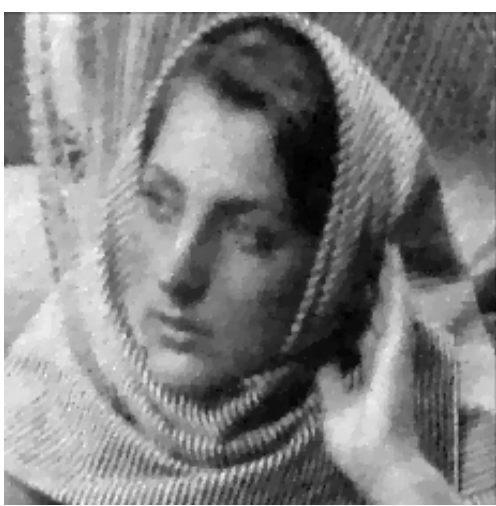

global TV (ROF)

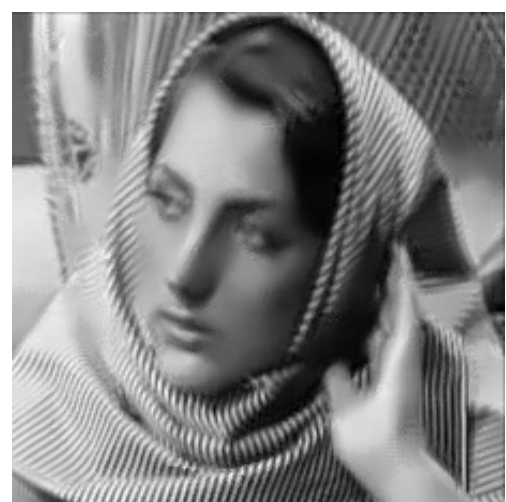

TV-means $(11 \times 11)$

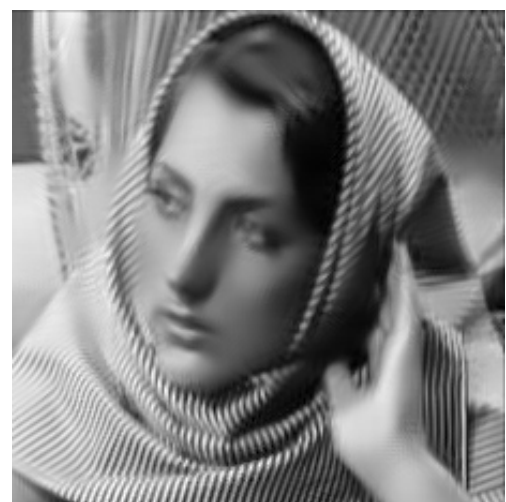

aggregated TV-means $(11 \times 11)$

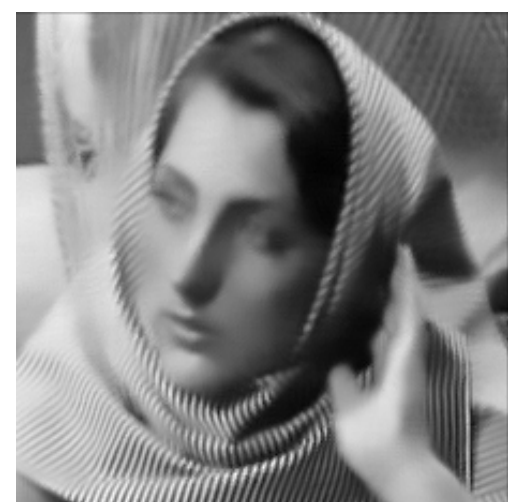

NL-means $(11 \times 11)$

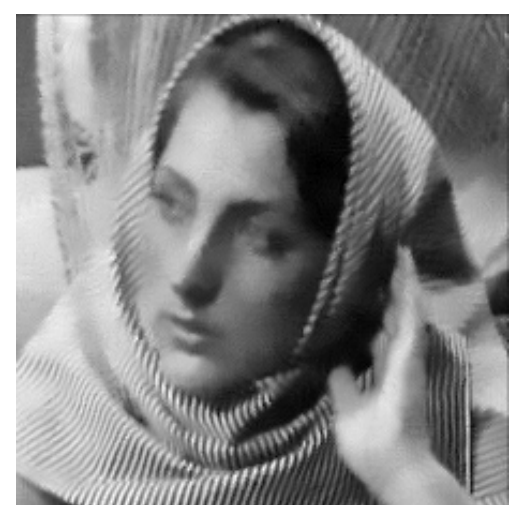

NL-means $(7 \times 7)$

Figure 7: Denoising of Barbara image (detail). The classic Barbara image, corrupted with a Gaussian white noise with standard deviation $\sigma=20$ (top, left), is denoised using several methods compared in this paper. The ROF method (bottom, left) does not manage to handle well the smooth and textured parts: most textures parts (scarf, basket chair) are poorly reconstructed, while a non-negligible amount of noise remains in the originally smooth regions (cheek, hand). The NL-means method (right column, top and bottom rows) performs better, but some regions are oversmoothed (eye, basket chair) due to the patch-jittering effect mentioned in the end of Section 7.1. This effect is particularly strong for $11 \times 11$ patches (top row); it can be reduced by using smaller patches $(7 \times 7$ on bottom row), which leads to a better image but with a noticeable loss of denoising in some parts (hand, cheek). The TV-means algorithm we propose (middle column) manages to find an interesting compromise: it avoids the patch-jittering effect without reducing too much the amount of denoising in other regions, thanks to the use of local TV-denoising. The aggregated version (middle column, bottom row) yields a balanced restoration, visually nicer and significantly better than other methods in terms of PSNR (see Table 1). Note that all images above were enhanced by a same affine contrast change for an easier visualization.

\section{Conclusion}

The aim of this work was to transform the well-known ROF $\left(\mathrm{TV}-L^{2}\right)$ filter into a local filter. This transformation is interesting for several reasons, and in particular because, as we showed, most image pixels have a very limited influence zone in the ROF model, so that such a local filter is expected to inherit the good behavior of the ROF model. We built a local TV-filter with an arbitrary neighborhood shape, and discovered that the introduction of an appropriate weight function (typically Gaussian) was necessary to avoid aliasing effects, which is quite intuitive considering the linear regime in which the local TV-filter falls for large values of the scale $\lambda$. Aside from interesting properties of this new local TV-filter (in particular a limiting PDE, allowing weight normalization), we established convergence conditions for the algorithms we built, inspired from Chambolle's previous works. We illustrated the interest of local 


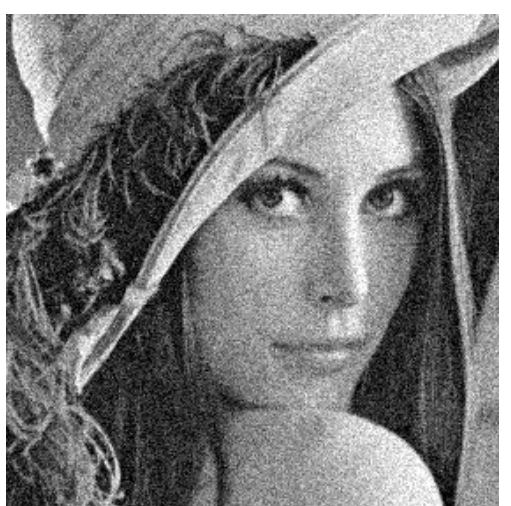

noisy

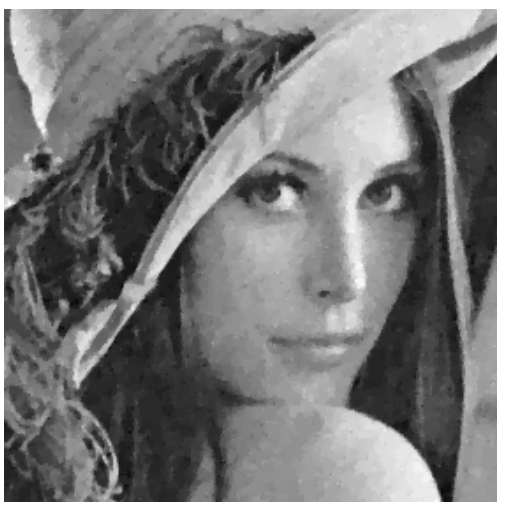

global TV $(\mathrm{ROF})$

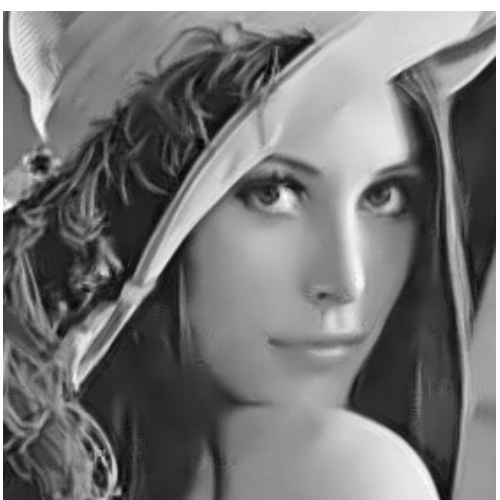

TV-means $(11 \times 11)$

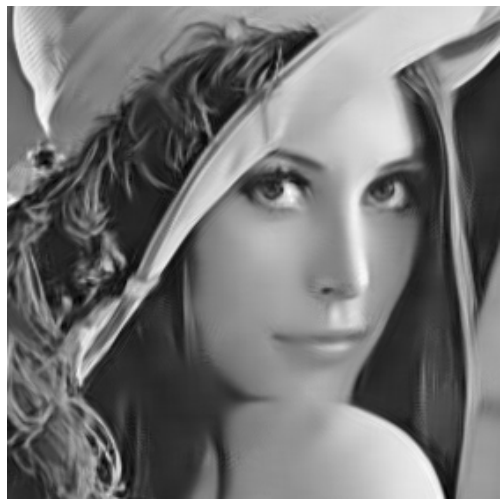

aggregated TV-means $(11 \times 11)$

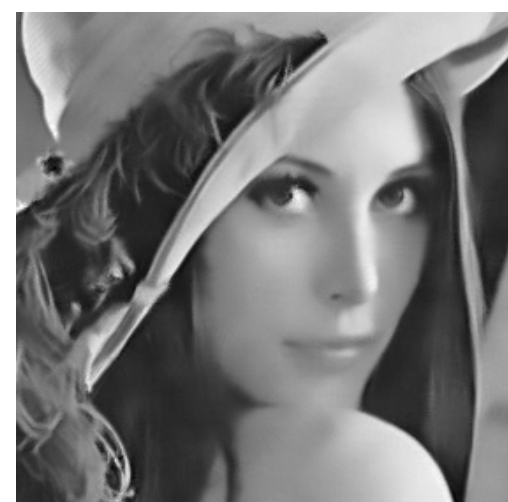

NL-means $(11 \times 11)$

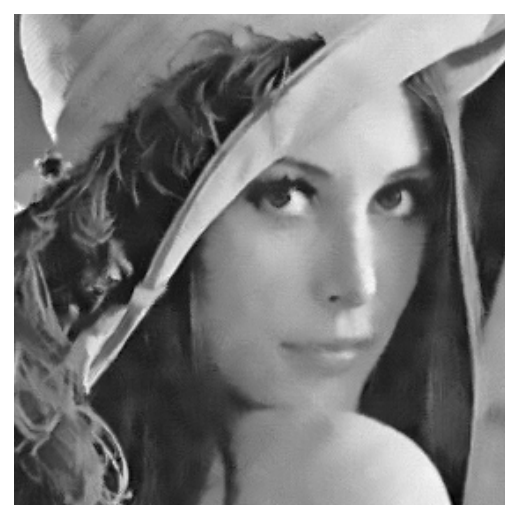

NL-means $(7 \times 7)$

Figure 8: Denoising of Lena image (detail). As in Figure 7, global TV-denoising simultaneously leaves noise (and staircasing) on the smooth parts and erases some texture (hat, feathers). The NL-means method performs better, but either leaves noticeable noise $(7 \times 7$ patches $)$, or introduces too much blur $(11 \times 11$ patches $)$. A more balanced treatment of edges, flat areas and textures is obtained with aggregated TV-means, yielding a better global impression and a more precise recovery of textures (see, e.g., the feathers and the thin stripes of the hat).

\begin{tabular}{|l|c|c|c|c|c|}
\cline { 2 - 6 } \multicolumn{1}{c|}{} & Barbara & Lena & Boats & House & Peppers \\
\multicolumn{1}{c|}{} & $512 \times 512$ & $512 \times 512$ & $512 \times 512$ & $256 \times 256$ & $256 \times 256$ \\
\hline noisy $(\sigma=20)$ & 22.1 & 22.1 & 22.1 & 22.1 & 22.1 \\
\hline TV $($ ROF) $[54]$ & 26.69 & 30.89 & 29.21 & 31.22 & 29.62 \\
\hline NL-means [15] & 29.59 & 31.50 & 29.32 & 32.05 & 30.12 \\
\hline TV-means & 29.94 & 31.80 & 29.34 & 32.34 & 29.73 \\
\hline agg. TV-means & $\mathbf{3 0 . 9 3}$ & $\mathbf{3 2 . 4 8}$ & $\mathbf{3 0 . 0 0}$ & $\mathbf{3 3 . 1 0}$ & $\mathbf{3 0 . 6 3}$ \\
\hline Kervrann et al. $[43]$ & 30.37 & 32.64 & 30.12 & 32.90 & 30.59 \\
\hline BM3D [31] & 31.78 & 33.05 & 30.88 & 33.77 & 31.29 \\
\hline
\end{tabular}

Table 1: PSNR values obtained with different denoising algorithms. We can observe that the aggregated TVmeans algorithm (in bold) shows similar performance than Kervrann et al. method, and systematically improves both TV and NL-means filtering. The simple combination of TV and NL-means we propose cannot compete directly with BM3D, but it could probably be integrated in a more sophisticated algorithm that could attain closer performances. 
TV-denoising in two directions. First, we showed that it brings an interesting bias-variance trade-off, compared to its linear regime (Gaussian filtering) and global TV-denoising. One illustration of this is the fantastic reduction of the well-known staircasing effect, that makes ROF-processed images look like oil paintings. Second, we used local TV-filtering to build a new filter that combines TV-denoising and NL-means into a simple but efficient denoising method called aggregated $T V$-means. This new filter brings interesting perspectives, in particular because it shows that although the ROF model is not any more a state-of-the-art denoising algorithm for most images, the idea of TV-denoising can still be used to build efficient denoising filters.

\section{Acknowledgements}

We thank Antonin Chambolle for fruitful discussions.

\section{Appendix: proof of Proposition 6}

We follow Chambolle's proof [21] and generalize it to the case of a non-rectangular and weighted domain.

1) First, we prove that the minimizer $u \in \mathbb{R}^{\mathcal{W}}$ of $E_{\lambda, \omega, v}(u)=\left\|D^{1 / 2}(u-v)\right\|^{2}+\lambda T V(u)$ (Equation 43) satisfies $u=v-\pi_{\frac{\lambda}{2} K}(v)$. Euler's equation for the minimization of $E_{\lambda, \omega, v}(u)$ writes

$$
2 D(u-v)+\lambda \partial T V(u) \ni 0,
$$

that is,

$$
\frac{D(v-u)}{\lambda / 2} \in \partial T V(u)
$$

Denoting $T V^{*}$ the Legendre-Fenchel transform of $T V$, we get, thanks to the Moreau decomposition (see $[41])$,

$$
u \in \partial T V^{*}\left(\frac{D(v-u)}{\lambda / 2}\right) .
$$

Now setting $w=\frac{v-u}{\lambda / 2}$, this yields

$$
w-\frac{2}{\lambda} v+\frac{2}{\lambda} \partial T V^{*}(D w) \ni 0
$$

whose left-hand term is the subdifferential of the energy $\tilde{E}_{\lambda, \omega, v}(w)=\frac{1}{2}\left\|w-\frac{2}{\lambda} v\right\|^{2}+\frac{2}{\lambda} T V^{*}(D w)$, and (62) implies that $w$ minimizes $\tilde{E}_{\lambda, \omega, v}$. Moreover, if $K$ is defined by (46), which makes sense since $D$ is invertible, from [21] we have

$$
T V^{*}(D w)= \begin{cases}0 & \text { if } w \in K \\ +\infty & \text { else, }\end{cases}
$$

which holds whatever the domain $\mathcal{W}$ as the operators $\nabla$ and div are dual one to the other. Hence the minimization of $\tilde{E}_{\lambda, \omega, v}$ amounts to project $v$ onto $\frac{\lambda}{2} K$, which is closed and convex because $p \mapsto D^{-1} p$ is linear. This precisely writes $u=v-\pi_{\frac{\lambda}{2} K}(v)$.

2) Recalling the definition of $K$ (Equation 46), step 1) enables us to write $w=D^{-1} \operatorname{div} p$, where $p$ minimizes $\left\|\frac{2}{\lambda} v-D^{-1} \operatorname{div} p\right\|^{2}$ among all $p$ such that $\|p\|_{\infty} \leq 1$. The necessary and sufficient KarushKuhn-Tucker conditions hold and there exists $\alpha \in \mathbb{R}^{\mathcal{W}}$ such that

$$
\forall x \in \mathcal{W}, \quad-\nabla\left(\frac{\lambda}{2} D^{-1} \operatorname{div} p-v\right)_{x}=\alpha_{x} p_{x}
$$

with $\alpha_{x} \geq 0$ and $\alpha_{x}\left(\left|p_{x}\right|^{2}-1\right)=0$ for all $x \in \mathcal{W}$. Then either $\alpha_{x}>0$ and $\left|p_{x}\right|=1$, or $\left|p_{x}\right|<1$ and $\alpha_{x}=0$. In both cases $\alpha_{x}=\left|\nabla\left(\frac{\lambda}{2} D^{-1} \operatorname{div} p-v\right)\right|_{x}$, and replacing this value of $\alpha_{x}$ in (63) concludes the proof of (47). 


\section{References}

[1] G. Acosta, R. G. Duràn, An optimal Poincaré inequality in $L^{1}$ for convex domains, Proc. Amer. Math. Soc., vol. 132, n. 1, pp. 195-202 (electronic), 2004.

[2] A. Almansa, C. Ballester, V. Caselles, G. Haro, A TV based restoration model with local constraints, J. Sci. Comput., vol. 34, n. 3, pp. 209-236, 2008.

[3] F. Alter, V. Caselles and A. Chambolle, Evolution of characteristic functions of convex sets in the plane by the minimizing total variation flow, Interfaces and Free Boundaries. Mathematical Modelling, Analysis and Computation, vol. 7, n. 1, pp. 29-53, 2005.

[4] F. Alter, V. Caselles and A. Chambolle, A characterization of convex calibrable sets in $\mathbb{R}^{n}$, Math. Ann., vol. 332, n. 2, pp. 329-366, June 2005.

[5] L. Alvarez, F. Guichard, P.-L. Lions and J.-M. Morel, Axioms and fundamental equations of image processing, Archive for Rational Mechanics and Analysis, vol. 123, n. 3, pp. 199-257, 1993.

[6] F. Andreu, V. Caselles, J.I. Diaz, J.M. Mazon, Some qualitative properties for the total variation flow. J. Func. Anal. 188, n. 2, pp. 516-547, 2002.

[7] L. Ambrosio, N. Fusco, D. Pallara, Functions of bounded variation and free discontinuity problems, Oxford University Press, March 2000.

[8] J.-F. Aujol, G. Aubert, L. Blanc-Féraud, A. Chambolle, Image decomposition into a bounded variation component and an oscillating component, J. Math. Imaging Vis., vol. 22, n. 1, pp. 71-88, 2005.

[9] J.-F. Aujol, Some first-order algorithms for total-variation based image restoration, J. Math. Imaging Vision, vol. 34, n. 3, pp. 307-327, July 2009.

[10] G. Bellettini, V. Caselles and M. Novaga, The total variation flow in $\mathbb{R}^{N}$, J. Differential Equations, vol. 184, n. 2, pp. 475-525, 2002.

[11] M. Bertalmio, V. Caselles, B. Rougé, A. Solé, A TV based restoration model with local constraints, J. Sci. Comput., vol. 19, n. 1-3, pp. 95-122, 2003.

[12] J. Besag, On the statistical analysis of dirty pictures, J. Roy. Statist. Soc. Ser. B, vol. 48, n. 3, pp. 259-302, 1986.

[13] P. Blomgren, T.F. Chan, P. Mulet, C. Wong, Total variation image restoration: numerical methods and extensions, IEEE Trans. Image Process., vol. 3, pp. 384-387, 1997.

[14] T. Brox, D. Cremers, Iterated nonlocal means for texture restoration, Scale-Space and Variational Methods, pp. 13-24, 2007.

[15] A. Buades, B. Coll and J.-M. Morel, A review of image denoising algorithms, with a new one, Multiscale Model. Simul., vol. 4, n. 2, pp. 490-530 (electronic), 2005.

[16] A. Buades, B. Coll and J.-M. Morel, The staircasing effect in neighborhood filters and its solution, IEEE Trans. Image Process., vol. 15, n. 6, pp. 1499-1595, 2006.

[17] J. Carter, Dual methods for total-variation based image restoration, Ph.D. thesis, U.C.L.A., 2001.

[18] V. Caselles and A. Chambolle, Anisotropic curvature-driven flow of convex sets, Nonlinear Analysis, vol. 65, n. $8,2006$.

[19] V. Caselles, A. Chambolle and M. Novaga, The discontinuity set of solutions of the TV denoising problem and some extensions, Multiscale Model. Simul., vol. 6, n. 3, pp. 879-894, 2007. 
[20] A. Chambolle, P.-L. Lions, Image recovery via total variation minimization and related problems, Numer. Math., vol. 76, pp. 167-188, 1997.

[21] A. Chambolle, An algorithm for total variation minimization and applications, J. Math. Imaging Vision, vol. 20, n. 1-2, pp. 89-97, January-March 2004.

[22] A. Chambolle, Total variation minimization and a class of binary MRF models, Proc. Energy Minimization Methods - Computer Vision and Pattern Recognition, pp. 136-152, 2005.

[23] T. Chan and C. Wong, Total variation blind deconvolution, IEEE Trans. Image Process., vol. 7, n. 3, pp. 370-375, 1998.

[24] T. Chan, A. Marquina, P. Mulet, High-order total variation-based image restoration, SIAM J. Sci. Comput., vol. 22, n. 2, pp. 503-516, 2000.

[25] T. Chan, A. Yip, F. Park, Simultaneous total variation image inpainting and blind deconvolution, Int. J. Imaging Systems and Technology, vol. 15, n. 1, pp. 92-102, 2005.

[26] T. Chan, J. Shen, H.-M. Zhou, Total variation wavelet inpainting, J. Math. Imaging Vision, vol. 25, n. 1, pp. 107-125, 2006.

[27] T. F. Chan, S. Esedoglu and F. E. Park, Image decomposition combining staircase reduction and texture extraction, J. Vis. Commun. Image Represent., vol. 18, n. 6, pp. 464-486, 2007.

[28] P. Chatterjee, P. Milanfar, Clustering-based denoising with locally learned dictionaries (K-LLD), IEEE Trans. Image Process., vol. 18, no. 7, pp. 1548-1451, July 2009.

[29] A. Criminisi, P. Perez, K. Toyama, Region filling and object removal by examplar-based image inpainting, IEEE Trans. Image Process., vol. 13, n. 9, pp. 1200-1212, 2004.

[30] P.-L. Combettes and V. R. Wajs, Signal Recovery by proximal forward-backward splitting, Multiscale Model. Simul., vol. 4, no. 4, pp. 1168-1200, November 2005.

[31] K. Dabov, A. Foi, V. Katkovnik, and K. Egiazarian, Image denoising by sparse 3D transform-domain collaborative filtering, IEEE Trans. Image Process., vol. 16, no. 8, August 2007.

[32] K. Dabov, A. Foi, V. Katkovnik, and K. Egiazarian, BM3D image denoising with shape-adaptive principal component analysis, Proc. Workshop on Signal Processing with Adaptive Sparse Structured Representations (SPARS'09), France, April 2009.

[33] J. Darbon and M. Sigelle, A fast and exact algorithm for total variation minimization, Trans. Iberian Conference on Pattern Recognition and Image Analysis vol. 1, pp. 351-359, 2005.

[34] J. Darbon and M. Sigelle, Image restoration with discrete constrained total variation. Part I: Fast and exact optimization, J. Math. Imaging Vision, vol. 26, n. 3, pp. 261-276, 2006.

[35] D. C. Dobson and F. Santosa, Recovery of blocky images from noisy and blurred data, Journal on Applied Mathematics, vol. 56, n. 4, pp. 1181-1198, 1996.

[36] S. Durand, M. Nikolova, Denoising of frame coefficients using $l^{1}$ data-fidelity term and edgepreserving regularization, Multiscale Model. Simul., vol. 6, n. 2, pp. 547-576 (electronic), 2007.

[37] A. A. Efros, T. K. Leung, Texture synthesis by non-parametric sampling, IEEE Int. Conf. on Comput. Vis., 1999.

[38] L. C. Evans and R. F. Gariepy, Measure theory and fine properties of functions, Studies in Advanced Mathematics, CRC Press, 1992.

[39] E. Fisher, A Skorohod representation and an invariance principle for sums of weighted i.i.d. random variables, Rocky Mountains J. Math., vol. 22, pp. 169-179, 1992. 
[40] F. Guichard and F. Malgouyres, Total variation based interpolation, Proc. European Signal Processing Conference, vol. 3, pp. 1741-1744, 1998.

[41] J.-B. Hiriart-Urruty and C. Lemaréchal, Convex analysis and minimization algorithms: A review of PDE models in image image processing and image analysis, in Grundlehren der Mathematischen Wissenshaft [Fundamental Principles of Mathematical Sciences], pp. 305-306, 1993, Springer Verlag.

[42] V. Katkovnik, A. Foi, K. Egiazarian, J. Astola, Directional varying scale approximations for anisotropic signal processing, Proc. European Signal Processing Conference, pp. 101-104, 2004.

[43] C. Kervrann, J. Boulanger, Local adaptivity to variable smoothness for exemplar-based image regularization and representation, Int. J. Computer Vision, vol. 79, n. 1, pp. 45-69, 2008.

[44] S. Levine, Y. Chen and J. Stanich, Image restoration via nonstandard diffusion, Technical Report n. 04-01, Dept mathematics and computer science, Duquesne University, 2004.

[45] C. Louchet, L. Moisan, Total variation denoising using posterior expectation, Proc. European Signal Processing Conference (electronic), August 2008.

[46] J. Mairal, F. Bach, J. Ponce, G. Sapiro, A. Zisserman, Non-local sparse models for image restoration, IEEE Int. Conf. on Computer Vision, 2009.

[47] F. Malgouyres, Rank related properties for basis pursuit and total variation regularization, Signal Processing, vol. 87, n. 11, pp. 2695-2707, 2007.

[48] M. Nikolova, Local strong homogeneity of a regularized estimator, SIAM J. Appl. Math., vol. 61, n. 2, pp. 633-658, 2000.

[49] M. Nikolova, Weakly constrained minimization : Application to the estimation of images and signals involving constant regions, J. Math. Imaging Vision, vol. 21, n. 2, pp. 155-175, 2004.

[50] J. Orchard, M. Ebrahimi, A. Wong, Efficient nonlocal-means denoising using the SVD, Int. Conf. on Image Processing, San Diego, California, USA, pp. 1732-1735, 2008.

[51] S. Osher, A. Solé, L. Vese, Image decomposition and restoration using total variation minimization and the $H^{-1}$ norm, Multiscale Model. Simul., vol. 1, n. 3, pp. 349-370, 2003.

[52] P. Perona, J. Malik, Scale-space and edge detection using anisotropic diffusion, IEEE Trans. on Pattern Analysis and Machine Intelligence, vol. 12, n. 7, pp. 629-639, 1990.

[53] W. Ring, Structural properties of solutions of total variation regularization problems, ESAIM, Math. Modelling and Numerical Analysis, vol. 34, pp. 799-840, 2000.

[54] L. Rudin, S. Osher, E. Fatemi, Nonlinear total variation based noise removal algorithms, Phys. D, vol. 60, n. 1-4, pp. 259-268, 1992.

[55] L. Rudin, S. Osher, Total variation based image restoration with free local constraints, IEEE Trans. Image Process., vol. 1, pp. 31-35, 1994.

[56] S.M. Smith and J. M. Brady, SUSAN-A new approach to low level image processing, Int. J. Comput. Vision, vol. 23, n. 1, pp. 45-78, 1997.

[57] D. M. Strong and T.F. Chan, Exact solutions to total variation regularization problems, UCLA CAM Report n. 96-41, 1996.

[58] C. Tomasi and R. Manduchi, Bilateral filtering for gray and color images, Proc. Int. Conf. Computer Vision, pp. 839-846, 1998.

[59] P. Weiss, L. Blanc-Féraud, G. Aubert, Efficient schemes for total variation minimization under constraints in image processing, SIAM J. Sci. Comput., vol. 31, n. 3, pp. 2047-2080, 2009.

[60] L. Yaroslavsky, M. Eden, Digital picture processing - an introduction, New-York, Springer-Verlag, 1985. 